Article

\title{
Revisiting a Negative Cosmological Constant from Low-Redshift Data
}

\author{
Luca Visinelli ${ }^{1,2, *}$, Sunny Vagnozzi ${ }^{2,3,4, *}$ and Ulf Danielsson ${ }^{1, *}$ \\ 1 Department of Physics and Astronomy, Uppsala University, Lägerhyddsvägen 1, SE-751 20 Uppsala, Sweden \\ 2 Nordita, KTH Royal Institute of Technology and Stockholm University, Roslagstullsbacken 23, \\ SE-106 91 Stockholm, Sweden \\ 3 The Oskar Klein Centre for Cosmoparticle Physics, Department of Physics, Stockholm University, \\ AlbaNova Universitetscentrum, Roslagstullsbacken 21A, SE-106 91 Stockholm, Sweden \\ 4 Kavli Institute for Cosmology (KICC) and Institute of Astronomy, University of Cambridge, \\ Madingley Road, Cambridge CB3 0HA, UK \\ * Correspondence: luca.visinelli@physics.uu.se (L.V.); sunny.vagnozzi@fysik.su.se (S.V.); \\ ulf.danielsson@physics.uu.se (U.D.)
}

Received: 18 July 2019; Accepted: 8 August 2019; Published: 10 August 2019

\begin{abstract}
Persisting tensions between high-redshift and low-redshift cosmological observations suggest the dark energy sector of the Universe might be more complex than the positive cosmological constant of the $\Lambda \mathrm{CDM}$ model. Motivated by string theory, wherein symmetry considerations make consistent AdS backgrounds (i.e., maximally-symmetric spacetimes with a negative cosmological constant) ubiquitous, we explore a scenario where the dark energy sector consists of two components: a negative cosmological constant, with a dark energy component with equation of state $w_{\phi}$ on top. We test the consistency of the model against low-redshift baryon acoustic oscillation and Type Ia supernovae distance measurements, assessing two alternative choices of distance anchors: the sound horizon at baryon drag determined by the Planck collaboration and the Hubble constant determined by the SHOES program. We find no evidence for a negative cosmological constant and mild indications for an effective phantom dark energy component on top. A model comparison analysis reveals that the $\Lambda \mathrm{CDM}$ model is favoured over our negative cosmological constant model. While our results are inconclusive, should low-redshift tensions persist with future data, it would be worth reconsidering and further refining our toy negative cosmological constant model by considering realistic string constructions.
\end{abstract}

Keywords: negative cosmological constant; $H_{0}$ tension; dark energy; low-redshift data; extended cosmological models

\section{Introduction}

While extremely successful at describing a wide variety of high- and low-redshift observations [1-5], the $\Lambda \mathrm{CDM}$ model has recently begun to display a number of small cracks $[6,7]$. One of the most tantalizing among these crevices is the so-called " $H_{0}$ tension", referring to the discrepancy between two independent estimates of the Hubble constant $H_{0}$. The first is a local estimate from the Hubble Space Telescope (HST), based on a distance ladder approach using Cepheids variables in the hosts of Type Ia Supernovae (SNeIa), yielding $H_{0}=(74.03 \pm 1.42) \mathrm{km} \mathrm{s}^{-1} \mathrm{Mpc}^{-1}$ [8]. The second is an indirect estimate based on Cosmic Microwave Background (CMB) temperature and polarization anisotropies measurements from the Planck collaboration: assuming an underlying $\Lambda \mathrm{CDM}$ model, a value of $H_{0}=(67.36 \pm 0.54) \mathrm{km} \mathrm{s}^{-1} \mathrm{Mpc}^{-1}$ [5] is obtained. Besides this cosmological tension, the $\Lambda \mathrm{CDM}$ model also suffers from a number of astrophysical shortcomings at small (galactic 
or subgalactic) scales, mostly referring to mismatches between simulations of cold dark matter and observations. Such issues include the core-cusp problem [9], the missing satellites problem [10] (although, see for example [11,12]), and the too-big-to-fail problem [13]. See for instance [14-16] for recent reviews on the subject. It has been speculated that such issues might signal the need to move away from the collisionless cold dark matter paradigm, and in particular that allowing for interactions between dark matter particles, or between dark matter and baryons, could alleviate these issues (see, e.g., [17-31] for an incomplete list of proposed models).

The statistical significance of the $H_{0}$ tension, currently at a level $\gtrsim 4 \sigma$, has steadily grown over recent years, owing also to improvements in the distance ladder approach [32-35]. Additional (re)analyses of the local distance ladder [36-46], as well as independent estimates of $H_{0}$ from strong-lensing time delays [47-49] have independently supported the conclusion that local measurements favour a higher value of $H_{0}$ [8] (see, however, the study in [50]). Given that the Planck estimate of $H_{0}$ relies on the assumption of an underlying $\Lambda \mathrm{CDM}$ model, it is possible that the $H_{0}$ tension might be hinting towards new physics, possibly involving non-standard properties of dark matter or dark energy [51-54]. The task is not easy to accomplish, since the simplest single-field quintessence models cannot solve the $H_{0}$ tension [55,56], even when a late-time transition from a matter-like Equation of State (EoS) is considered [57]. For an incomplete list of works in this direction, see $[55,57-141]$.

Measurements of temperature and polarization anisotropies in the CMB exquisitely constrain $\theta_{s}$, the angular scale of the sound horizon at last-scattering:

$$
\theta_{s}=\frac{r_{s}\left(z_{\mathrm{drag}}\right)}{D_{A}\left(z_{\star}\right)}
$$

where $z_{\star} \approx 1100$ is the redshift of last-scattering, $z_{\text {drag }} \approx z_{\star}$ is the redshift of the drag epoch (when baryons are released from the Compton drag of photons), $r_{s}(z)$ is the comoving sound horizon at redshift $z$, and $D_{A}(z)$ is the angular diameter distance to redshift $z$. Attempts to address the $H_{0}$ tension by introducing new physics generally modify either or both $r_{s}$ and $D_{A}$, in such a way that an increase in $H_{0}$ is required in order to keep $\theta_{s}$ fixed. One of the simplest possibilities is to modify the Dark Energy (DE) sector in order to lower the expansion rate at late times, while leaving the early-time expansion rate unaltered. Such a change will keep $r_{s}\left(z_{\text {drag }}\right)$ unchanged, but will raise $D_{A}\left(z_{\star}\right)$. In order to keep $\theta_{s}$ fixed, a higher value of $H_{0}$ will be inferred.

One of the simplest modifications to the DE sector that could be considered consists of replacing the positive cosmological constant (whose equation of state is $w_{\mathrm{cc}}=-1$ ) with a quintessence field with a time-varying EoS. A single minimally-coupled quintessence field $\phi$ with a standard kinetic term possesses a positive energy density with an equation of state $w_{\phi}(z) \geq-1$; it is easy to show that, in this case, the expansion rate actually increases at late times, going in the opposite direction of what is required to address the $H_{0}$ tension [55]. In fact, insisting on a positive energy density for the DE component brings one to consider phantom DE, i.e., a DE component with equation of state $w_{\phi}<-1$. Phantom DE components, which generically predict the Universe to end in a Big Rip [142] (although this fate can be avoided in certain classes of modified gravity theories [143-147]), are however generically extremely challenging to construct, due to the violation of the strong energy condition. Nonetheless, attempts to realize effectively stable phantom DE components (for instance within modified gravity or brane-world models) exist; see, e.g., [148-162].

The situation changes if one chooses not to restrict to the DE energy density being positive. Recent work has in fact shown that a number of persisting low-redshift tensions (including the $H_{0}$ tension) might be addressed if one allows for an evolving DE component whose energy density can assume negative values (see, e.g., [53,93,163-165]). In particular, the work in [165] considered a very interesting case, where the $\mathrm{DE}$ sector consists of a slowly-rolling quintessence field (whose energy density is positive) on top of a negative cosmological constant. Such a scenario is extremely interesting from a string theory perspective. In fact, constructing meta-stable de Sitter (dS) vacua 
(i.e., with a positive cosmological constant) has notoriously been a daunting task in string theory (see, e.g., [166-175]). These difficulties have led to the suggestion that string theory might not have any dS vacua at all [176-180], an observation that, if correct, would have interesting consequences for cosmology [181-190] (see, however, also the important work of [191], which appears to provide a possible counterexample to these swampland conjectures). In contrast, a negative cosmological constant (providing an AdS background, i.e., a maximally-symmetric spacetime with a negative cosmological constant) is very natural from symmetry considerations in string theory, as can be argued using the AdS/CFTcorrespondence [192]. Contrary to the case of dS, there does seem to exist a large number of consistent AdS backgrounds that can be obtained from string theory (it has even been suggested that AdS vacua can lead to an accelerating Universe [193]).

Another rather generic prediction of string theory is the plethora of light bosons known as the "axiverse". These correspond to moduli determining the size and shape of the extra dimensions and could give rise to important observable consequences that might alter the evolution of the Universe between recombination and today [194-208]. It is therefore interesting to try matching cosmological data using quintessence (possibly with an effective phantom equation of state, given that one is anyhow not dealing with a single quintessence field) in combination with a negative cosmological constant, both of which are natural from the point of view of string theory. This is an important motivation for our paper.

At any rate, determining the sign of a possible cosmological constant is crucial in order to understand the structure and fabric of space-time itself. In this work, we revisit the possibility that a negative cosmological constant might be allowed by current cosmological data, and more generally explore whether the $H_{0}$ tension might be relaxed within such a scenario. We first consider the standard $\Lambda \mathrm{CDM}$ model (featuring a positive cosmological constant with equation of state $w=-1$ ). We then move on to one of the simplest extension of the $\Lambda$ CDM model, i.e., the $w \mathrm{CDM}$ model, wherein the DE $\operatorname{EoS} w$ is treated as a free parameter. Finally, we consider a scenario in which the present accelerated expansion rate is driven by a combination of a cosmological constant whose density parameter $\Omega_{c c}$ is strictly negative, on top of which we allow for a second DE component with EoS $w_{\phi}$ (which can take on values $w_{\phi}<-1$ ) and a positive density parameter $\Omega_{\phi}$. While the case for a negative cosmological constant is clearly inspired by a string scenario, the present accelerated expansion rate requires that $\Omega_{\mathrm{cc}}+\Omega_{\phi}>0$. We refer to this last model as $c$ CDM. Notice that the requirement $\Omega_{\mathrm{cc}}+\Omega_{\phi}>0$, necessary in order for the Universe to accelerate, prevents us from considering a model where the dark energy sector consists solely of a negative cosmological constant, without the quintessence field on top.

In this work, we perform a Markov Chain Monte Carlo (MCMC) analysis to compare the three models (in order of increasing complexity $\Lambda \mathrm{CDM}, w \mathrm{CDM}$, and $c \mathrm{CDM}$ ) in light of recent low-redshift $(z \lesssim 2.5)$ distance measurements from a selection of Baryon Acoustic Oscillation (BAO) surveys [3,209-213] and Type Ia Supernovae (SNeIa) from the Pantheon catalogue [214]. The interpretation of BAO measurements requires "anchoring" them to either the cosmic distance ladder through independent measurements of $H_{0}$, or to the inverse distance ladder through independent measurements of $r_{\mathrm{drag}}$, the sound horizon at baryon drag [51]. To show the impact of the $H_{0}$ tension, we choose two different anchors to interpret our BAO data: we first use the local distance ladder measurement of $H_{0}$ from the "Supernovae and $H_{0}$ for the Dark Energy Equation of State" (SH0ES) program [8], before comparing our results to those obtained anchoring to the most recent measurement of $r_{\text {drag }}$ from the Planck collaboration [5]. More details on the datasets chosen and the choice of anchoring are given in Section 2.

Our treatment of the effective DE component on top of the negative cosmological constant is purely phenomenological, and we remain agnostic as to the underlying Lagrangian for such a component (see, e.g., $[215,216]$ for early works discussing a fundamental origin for this type of composite dark energy). While we envisage such a component being a quintessence field slowly rolling along a potential on top of the negative cosmological constant, we allow the effective equation of state $w_{\phi}$ to enter the phantom regime, where $w_{\phi}<-1$. The rationale is that string constructions generically predict 
a large number of light bosons on top of the stable AdS vacua: therefore, one can in general be faced with a multi-field quintessence scenario, whose effective behaviour might be phantom (see, e.g., [217-221]) (other possibilities for obtaining an effective phantom component from an underlying scalar field model involve considering modifications to gravity [222-231], couplings between dark energy and dark matter [232-246], particle creation mechanisms [247-256], or invoking scalar fields non-minimally coupled to gravity or with a kinetic term that is non-canonical [257-261]). In the cCDM model, the quintessence field (whose effective $\operatorname{EoS} w_{\phi}$ we allow to be phantom, again from a purely effective perspective) is treated as an additional contribution to the total energy density along with the (possibly negative) cosmological constant and the usual components of matter and radiation. Our results are interesting from a model-building perspective, as we find that from the low-redshift data side, a negative cosmological constant is a valid alternative to a phantom dark energy component.

The rest of the paper is then organized as follows. In Section 2, we describe the datasets we use. In Section 3, we describe in detail the models considered along with their parameter spaces. The MCMC analysis performed in order to constrain the models, as well as the obtained results are described in Section 4. We provide concluding remarks in Section 5.

\section{Overview of the Datasets Used}

The evolution of the Hubble expansion rate on the redshift $z, H(z)$, is usually factored out as $H(z)=H_{0} E(z)$, where $H_{0}$ is the Hubble constant and the normalized expansion rate $E(z)$ is a dimensionless function describing the evolution of the expansion rate with the redshift. Let us introduce the angular diameter distance to redshift $z, D_{A}(z)$, given by the following:

$$
D_{A}(z) \equiv \frac{1}{1+z} \frac{c}{H_{0}} \int_{0}^{z} \frac{d z^{\prime}}{E\left(z^{\prime}\right)}
$$

The angular diameter distance in Equation (2) is related to the comoving angular diameter distance to redshift $z, D_{M}(z)$, by $D_{A}(z)=D_{M}(z) /(1+z)$. Let us further define the sound horizon at redshift $z$, $r_{s}(z)$, as the distance travelled by an acoustic wave in the baryon-photon plasma from a very early time, given by:

$$
r_{s}(z)=\int_{z}^{\infty} \frac{c_{s}\left(z^{\prime}\right)}{H\left(z^{\prime}\right)} d z^{\prime}
$$

where $c_{s}(z)$ is the sound speed of the baryon-photon plasma at redshift $z$.

We consider two classes of observational datasets. A first class consists of distance measurements from Baryon Baryon Acoustic Oscillations (BAO) surveys, as well as from Type Ia Supernovae (SNeIa). A second class consists of two different types of anchors used to interpret the BAO measurements. Specifically, we consider two different anchors: direct measurements of the Hubble constant based on the local Cepheids-Supernovae distance ladder and CMB measurements of the comoving sound horizon at baryon drag, further defined in Equation (4) below. We describe these four datasets in more detail below

- BAO: In the early Universe, the interplay between gravity and radiation pressure sets up acoustic oscillations, which produce a sharp feature in the two-point correlation function of luminous matter at a scale equal to the comoving size of the sound horizon at the drag epoch [262-266]:

$$
r_{\text {drag }} \equiv r_{s}\left(z_{\text {drag }}\right),
$$

where the drag epoch is the time when the baryons are released from the Compton drag of the photons, and occurs at a redshift $z_{\mathrm{drag}}$. Measurements of the BAO feature, first reported in $[265,267]$, are usually performed at an effective redshift $z_{\text {eff. }}$ These measurements can in principle independently constrain the angular diameter distance $D_{A}\left(z_{\text {eff }}\right)$ in units of $r_{\text {drag }}$ (for modes in the transverse direction with respect to the line of sight) and $H\left(z_{\text {eff }}\right) r_{\text {drag }}$ (for modes 
along the line of sight), whereas isotropic $\mathrm{BAO}$ measurements constrain a combination of these quantities known as the volume distance $D_{V}\left(z_{\text {eff }}\right)$. Here, we consider various types of BAO measurements, which constrain the following quantities:

$$
\begin{aligned}
& \frac{D_{M}\left(z_{\text {eff }}\right)}{r_{\text {drag }}} \equiv \frac{c}{H_{0} r_{\text {drag }}} \int_{0}^{z_{\text {eff }}} \frac{d z^{\prime}}{E\left(z^{\prime}\right)}, \\
& \frac{D_{H}\left(z_{\text {eff }}\right)}{r_{\text {drag }}} \equiv \frac{c}{H_{0} r_{\text {drag }}} \frac{1}{E\left(z_{\text {eff }}\right)}, \\
& \frac{D_{V}\left(z_{\text {eff }}\right)}{r_{\text {drag }}} \equiv \frac{c}{H_{0} r_{\text {drag }}} \sqrt[3]{\frac{z_{\text {eff }}}{E\left(z_{\text {eff }}\right)}\left(\int_{0}^{z_{\text {eff }}} \frac{d z^{\prime}}{E\left(z^{\prime}\right)}\right)^{2}},
\end{aligned}
$$

See Appendix B of [268] for a more detailed discussion of BAO measurements.

In our analysis, we consider anisotropic BAO measurements from the Baryon Oscillation Spectroscopic Survey (BOSS) collaboration [3] Data Release 12 (DR12) at the effective redshifts $z_{\text {eff }}=0.38,0.51,0.61$ and from Lyman- $\alpha$ forest samples at $z_{\text {eff }}=2.40[211,212]$. We also include isotropic BAO measurements from the Six-degree Field Galaxy Survey (6dFGS) at $z_{\text {eff }}=0.106$ [209], from the Sloan Digital Sky Survey Data (SDSS) Main Galaxy Sample (MGS) release at $z_{\text {eff }}=0.15$ [210] and from the quasar sample of the extended Baryon Oscillation Spectroscopic Survey (eBOSS) at $z_{\text {eff }}=1.52$ [213]. For completeness, we collect the BAO measurements used in this work in Table 1. We constructed the likelihood for the BAO data, $\mathcal{L}_{\mathrm{BAO}}$, using the data described and the correlation matrices provided by the collaborations.

Table 1. BAO scale measurements used in this work. For BOSS DR12, we set the fiducial scale $r_{\text {fid }}=147.78 \mathrm{Mpc}[3]$.

\begin{tabular}{cccc}
\hline Dataset & $z$ & Measurement & Reference \\
\hline BOSS DR12 & 0.38 & $H\left(r_{\text {drag }} / r_{\text {fid }}\right)=(81.2 \pm 2.2 \pm 1.0) \mathrm{km} /(\mathrm{s} \mathrm{Mpc})$ & {$[3]$} \\
BOSS DR12 & 0.51 & $H\left(r_{\text {drag }} / r_{\text {fid }}\right)=(90.9 \pm 2.1 \pm 1.1) \mathrm{km} /(\mathrm{s} \mathrm{Mpc})$ & {$[3]$} \\
BOSS DR12 & 0.61 & $H\left(r_{\text {drag }} / r_{\text {fid }}\right)=(99.0 \pm 2.2 \pm 1.2) \mathrm{km} /(\mathrm{s} \mathrm{Mpc})$ & {$[3]$} \\
BOSS DR12 & 0.38 & $D_{M} / r_{\text {drag }}=(1512 \pm 22 \pm 11) \mathrm{Mpc} / r_{\text {fid }}$ & {$[3]$} \\
BOSS DR12 & 0.51 & $D_{M} / r_{\text {drag }}=(1975 \pm 27 \pm 14) \mathrm{Mpc} / r_{\text {fid }}$ & {$[3]$} \\
BOSS DR12 & 0.61 & $D_{M} / r_{\text {drag }}=(2307 \pm 33 \pm 17) \mathrm{Mpc} / r_{\text {fid }}$ & {$[3]$} \\
BOSS DR12 + Ly $\alpha$ & 2.40 & $D_{M} / r_{\text {drag }}=36.6 \pm 1.2$ & {$[211]$} \\
BOSS DR12 + Ly $\alpha$ & 2.40 & $D_{H} / r_{\text {drag }}=8.94 \pm 0.22$ & {$[211]$} \\
6dFGS & 0.106 & $D_{V} / r_{\text {drag }}=2.98 \pm 0.13$ & {$[209]$} \\
SDSS-MGS & 0.15 & $D_{V} / r_{\text {drag }}=4.47 \pm 0.17$ & {$[210]$} \\
eBOSS quasars & 1.52 & $D_{V} / r_{\text {drag }}=26.1 \pm 1.1$ & {$[213]$} \\
\hline
\end{tabular}

- $\quad$ SNeIa: SNeIa catalogues report the corrected apparent magnitude of the $i$ th supernova, which is given by the following:

$$
\mu^{i}=M+5 \log _{10}\left(D_{A}^{i}(z) / \mathrm{Mpc}\right)+25,
$$

where $M$ is the absolute magnitude of the $i$ th supernova, which fixes the scale for the global fit. For practical purposes, we rewrite the relation as:

$$
\mu^{i}=5.7+5 \log _{10}\left(\frac{c(1+z)}{H_{0} \ell_{\mathrm{SN}}} \int_{0}^{z} \frac{d z^{\prime}}{E\left(z^{\prime}\right)}\right)
$$


where the absolute magnitude has been reabsorbed into a parameter $\ell_{\mathrm{SN}}=10^{-(M+19) / 5} \mathrm{Mpc}$, which we fit to data. We construct the likelihood:

$$
\mathcal{L}_{\mathrm{SN}}=\exp \left[-\frac{1}{2}(\mu-\mu \mathrm{obs})^{T} C_{\mathrm{SN}}^{-1}\left(\mu-\mu_{\mathrm{obs}}\right)\right],
$$

where the vector of apparent magnitude measurements $\mu_{\mathrm{obs}}$ and the corresponding covariance matrix $C_{\mathrm{SN}}^{-1}$ are taken from the Pantheon SNeIA dataset [214]. This catalogue provides luminosity distances $D_{A}(z)$ for 1048 SNeIa within the range $0.01<z \leq 2.3$ of Type 1a supernovae. Although the Pantheon analysis is marginally model-dependent, as biases in light-curve parameters are corrected assuming a $\Lambda \mathrm{CDM}$ cosmology, independent analyses run using the JLA SN dataset [269] have found that the model-dependence of the light-curve parameters is weak.

- Anchors: The cosmic distance ladder technique relies on measuring distances of extra-galactic objects, at distances beyond $\sim 100 \mathrm{Mpc}$, to map the Hubble flow. The expansion history of the Universe is mapped through Type 1a Supernovae (SNeIa) datasets [214,269-275] and BAO [265,267]. These distance scales need to be calibrated through anchors either at the high-redshift end (through $r_{\text {drag }}$ ) or at the low-redshift end (through $H_{0}$ ) [276].

For instance, $\mathrm{BAO}$ measurements constrain the combination $H_{0} r_{\mathrm{drag}}$. In fact, the tension between measurements at low and high redshifts of $H_{0}$ can be eased by modifying the value of $r_{\text {drag }}$, which is the standard ruler providing the BAO length scale. The sole measurements of either $H_{0}$ or $r_{\text {drag }}$ are respectively interpreted as anchoring the cosmic distance ladder or the inverse cosmic distance ladder. In any case, in order to interpret the BAO measurements, we have to anchor either $H_{0}$ or $r_{\text {drag }}$ to an independent evaluation [51]. Here, we consider independent approaches, namely calibrating the cosmic distance ladder by means of the recent measurements of $H_{0}$ in [8] or calibrating the inverse distance ladder by using the CMB measurement of $r_{\mathrm{drag}}$ in [5]. It is worth remarking that much (but not all) of the information contained in the CMB temperature and anisotropy spectra resides in the position and height of the first acoustic peak, which accurately constrains $\theta_{s}$ given in Equation (1) and provides valuable information about the geometry and the content of the Universe. The position of the first peak is sensitive to early-time modifications through a change in $r_{\mathrm{drag}}$, as well as to late-time modifications through a change in $D_{A}\left(z_{\star}\right)$.

Here, we consider two scenarios using two different anchors, given by the following:

1. Sound horizon at drag epoch (Scenario I): The anchor at the CMB epoch is expressed by the comoving sound horizon at the end of the baryon drag epoch $r_{\mathrm{drag}}$. Measurements of CMB temperature and polarization anisotropies by the Planck collaboration (PlanckTTTEEE + lowP dataset) give $r_{\text {drag }}=(147.05 \pm 0.30) \mathrm{Mpc}$ at a $68 \%$ Confidence Level (C.L.). When anchoring data to the sound horizon $r_{\text {drag, }}$, we construct the likelihood function $\mathcal{L}_{\text {Anchor }}=\mathcal{L}_{\text {drag }}$ as a Gaussian in $r_{\text {drag }}$ using this measurement.

2. Hubble constant $H_{0}$ (Scenario II): When anchoring data to the present value of the Hubble rate, we use the latest result from the SHOES program [8], which reports the local measurement $H_{0}=(74.03 \pm 1.42) \mathrm{km} \mathrm{s}^{-1} \mathrm{Mpc}^{-1}$. This measurement is used to construct the likelihood function $\mathcal{L}_{\text {Anchor }}=\mathcal{L}_{\text {Ceph }}$ as a Gaussian in $H_{0}$.

Since one of our main interests is to compare the results obtained using different anchors, we have not used other available datasets such as direct determinations of the Hubble rate $H(z)$ at redshifts $0.1 \lesssim z \lesssim 2[277,278]$ or "compressed" CMB likelihoods $[228,279,280]$. For the ease of comparison to related works, we chose to restrict our use of low-redshift data solely to BAO and SNe data, which are the two most robust low-redshift datasets widely used by the community. We did not include other late-time measurements of the expansion history, such as cosmic chronometers or distance measurements from Gamma-ray bursts. However, it would be interesting to further include these datasets, which could perhaps improve our constraints on possible negative cosmological constants, and we leave this exercise for future work. 


\section{Overview of Models and Model Comparison}

As described in the Introduction, we consider three models with increasing level of complexity to fit the data. For each model, $\rho_{i}$ represents the energy density in the species $i, \rho_{\text {crit }}=3 M_{\mathrm{PL}}^{2} H_{0}^{2}$, where $M_{\mathrm{PL}}$ is the reduced Planck mass, and we introduce the density parameter $\Omega_{i}=\rho_{i} / \rho_{\text {crit }}$. The present energy density in radiation $\Omega_{r}=5 \times 10^{-5}$ is obtained from the CMB temperature measured from the four-Year COBE-DMR CMB mission [281] as $T_{0}=(2.728 \pm 0.004) \mathrm{K}$ at $95 \%$ C.L. The fractional energy density in neutrinos $\Omega_{v}(z)$ assumes three active neutrinos of which two are massless and the third has a mass $m_{v_{3}}=0.06 \mathrm{eV}$, as done in the baseline Planck analyses. The value $0.06 \mathrm{eV}$ is the minimal neutrino mass sum allowed within the normal ordering [282], which is mildly favoured by current data [283-286]. Leaving the neutrino mass as a free parameter would not change our analysis substantially, given the current very tight upper limits on the sum of the neutrino masses $[5,283,287-293]$. We refer to the combination of invisible components as $\Omega_{\text {inv }}(z) \equiv \Omega_{r}(1+z)^{4}+\Omega_{v}(z)$. We treat baryons and CDM equivalently, both contributing to a total energy share $\Omega_{M}$. The remaining parameters are model-dependent and are discussed in the following.

1. $\Lambda \mathrm{CDM}$ model: Here, the normalized expansion rate is modelled as follows:

$$
E_{\Lambda \mathrm{CDM}}(z)=\left[\Omega_{\Lambda}+\Omega_{M}(1+z)^{3}+\Omega_{\mathrm{inv}}(z)\right]^{1 / 2} .
$$

We fix the value of $\Omega_{\Lambda}$ by demanding that $E_{\Lambda \mathrm{CDM}}(0)=1$, so that the parameter space associated with this model $\mathcal{S}_{\Lambda \mathrm{CDM}}=\left\{\Omega_{M}, H_{0}, r_{\mathrm{drag}}, \ell_{\mathrm{SN}}\right\}$ is four-dimensional.

2. $w \mathrm{CDM}$ model: Here, the normalized expansion rate is modelled as follows:

$$
E_{w \mathrm{CDM}}(z)=\left[\Omega_{\phi}(1+z)^{3\left(1+w_{\phi}\right)}+\Omega_{M}(1+z)^{3}+\Omega_{\mathrm{inv}}(z)\right]^{1 / 2},
$$

where $w_{\phi}$ is a constant. We fix the value of $\Omega_{\phi}$ by demanding that $E_{w \mathrm{CDM}}(0)=1$, so that the parameter space associated with this model $\mathcal{S}_{w \mathrm{CDM}}=\left\{\Omega_{M}, w_{\phi}, H_{0}, r_{\mathrm{drag}}, \ell_{\mathrm{SN}}\right\}$ is five-dimensional. Note that we allow $w_{\phi}$ to enter the phantom regime, where $w_{\phi}<-1$, since we are not restricting our attention to the case where the dark energy component is described by a single minimally-coupled quintessence field with a canonical kinetic term.

3. $c \mathrm{CDM}$ model: Here, the normalized expansion rate is modelled as follows:

$$
E_{c \mathrm{CDM}}(z)=\left[\Omega_{\mathrm{cc}}+\Omega_{\phi}(1+z)^{3\left(1+w_{\phi}\right)}+\Omega_{M}(1+z)^{3}+\Omega_{\mathrm{inv}}(z)\right]^{1 / 2} .
$$

We fix the value of $\Omega_{\phi}$ by demanding that $E_{w \mathrm{CDM}}(0)=1$, so that the parameter space associated with this model $\mathcal{S}_{\mathrm{cCDM}}=\left\{\Omega_{\mathrm{cc}}, \Omega_{M}, w_{\phi}, H_{0}, r_{\mathrm{drag}}, \ell_{\mathrm{SN}}\right\}$ is six-dimensional. We demand that $\Omega_{\mathrm{cc}}$ be strictly negative by exploring the region of the parameter space $\Omega_{\mathrm{cc}}<0$ when fitting the parameters in $\mathcal{S}_{c \mathrm{CDM}}$ against the data described in Section 2.

To sample the posterior distribution of the parameters, we perform a Markov Chain Monte Carlo (MCMC) analysis using the open-source Python package emcee [294]. For each model $\mathcal{M}$ described in Section 3, we explore the associated parameter space $\mathcal{S}_{\mathcal{M}}$ by performing the analysis with a Metropolis-Hastings algorithm and assuming flat priors for all variables, except for the anchor for which we assume a Gaussian prior with mean and standard deviation given by the corresponding measurement. In more detail, we assume a flat prior for $\Omega_{M} \in[0,1]$ and $\ell_{\mathrm{SN}} \in[0.9,1.2] \mathrm{Mpc}$ for all models discussed, in addition to the DE $\operatorname{EoS} w_{\phi} \in[-2,-0.5]$ for the $w \mathrm{CDM}$ and $c \mathrm{CDM}$ models and the cosmological constant $\Omega_{\mathrm{cc}} \in[-30,0]$ for the $c \mathrm{CDM}$ model. When anchoring the distance ladder to the comoving sound horizon at the drag epoch (Scenario I), we choose the flat prior $H_{0} \in$ $[60,80] \mathrm{km} / \mathrm{s} / \mathrm{Mpc}$ and a Gaussian prior for $r_{\text {drag }}$ based on the measurement by the Planck collaboration. When the present Hubble rate is used as the anchor (Scenario II), we choose the flat prior $r_{\text {drag }} \in$ 
$[120,160] \mathrm{Mpc}$ and a Gaussian prior for $H_{0}$ given by the SH0eS measurements [8]. We use the expression for the likelihood:

$$
\mathcal{L}=\mathcal{L}_{\mathrm{BAO}}+\mathcal{L}_{\mathrm{SN}}+\mathcal{L}_{\text {Anchor }}
$$

It is worth noting that the $\Lambda \mathrm{CDM}$ and $w \mathrm{CDM}$ models are nested models, i.e., the former is a particular case of the latter, recovered when setting $w=-1$. As such, we expect that the fit to data (quantified, e.g., through the $\chi^{2}$ ) should not worsen when moving from the $\Lambda \mathrm{CDM}$ to the $w \mathrm{CDM}$ model. However, the same is not true for the $c \mathrm{CDM}$ model. In fact, within the $c \mathrm{CDM}$ model, we require $\Omega_{c c}<0$, i.e., that the cosmological constant be strictly negative. Therefore, it is not possible to recover either the $\Lambda \mathrm{CDM}$ or the $w \mathrm{CDM}$ model as a particular limit of $c \mathrm{CDM}$. As a result, the fit to data will not necessarily improve when considering a negative cosmological constant.

At any rate, given that the $w \mathrm{CDM}$ and $c \mathrm{CDM}$ models possess respectively one and two extra parameters compared to the baseline $\Lambda \mathrm{CDM}$ model, it is important to assess whether the increased model complexity is warranted by a substantially better fit (if any) to the data. We performed a simple model comparison adopting the Akaike Information Criterion (AIC) to compare the competing models. The AIC of a given model is defined as [295]:

$$
\mathrm{AIC}=2 k+\min \left(\chi^{2}\right),
$$

where $k$ is the number of parameters of the model and $\min \left(\chi^{2}\right)$ is the $\chi^{2}$ calculated at the best-fit point of the model. Therefore, $k=4,5,6$ for the $\Lambda \mathrm{CDM}, w \mathrm{CDM}$, and $c \mathrm{CDM}$ models, respectively.

Assume we have two competing models $i$ and $j$, with $\mathrm{AIC}$ values $\mathrm{AIC}_{i}$ and $\mathrm{AIC}_{j}$, respectively, and assume that $\mathrm{AIC}_{i}<\mathrm{AIC}_{j}$. Then, model $i$ is to be preferred since it has a lower AIC. From Equation (15), we see that one needs an improvement in the fit of at least $\Delta \chi^{2} \geq 2 \Delta k$ in order to justify the increased number of parameters (for instance, $\Delta \chi^{2}=2$ when moving from $\Lambda C D M$ to $w \mathrm{CDM}$ or $\Delta \chi^{2}=4$ when moving to $\left.c \mathrm{CDM}\right)$. Moreover, the quantity $\exp \left[\left(\mathrm{AIC}_{i}-\mathrm{AIC}_{j}\right) / 2\right]$ gives the relative likelihood of model $i$ with respect to model $j$.

\section{Results}

We now discuss the results obtained analysing the datasets described in Section 2 within the context of the three models described in Section 3, which we then compare using the AIC described in the same section. In Table 2, we show corner plots visualizing the 1D marginalized and 2D joint posterior distributions for the parameters of the models considered: $\Lambda$ CDM model (upper panels), $w \mathrm{CDM}$ (middle panels), and $c \mathrm{CDM}$ model (lower panels), anchoring the BAO measurements either to $r_{\text {drag }}$ as measured by Planck (left column) or to $H_{0}$ as measured by SHOES (right column). Constraints on the parameters of the three models are also presented in Table 3.

As we see from Table 3, our analysis did not reveal any evidence for a non-zero negative cosmological constant. In fact, when analysing our datasets within the $c \mathrm{CDM}$ model, we only obtained a lower limit of $\Omega_{c c} \gtrsim-14$ regardless of whether we used $r_{\text {drag }}$ as measured by Planck or $H_{0}$ as measured by SHOES as the anchor. Moreover, within both the $w \mathrm{CDM}$ and $c$ CDM models, we saw a $1 \sigma$ preference for a phantom DE component $\left(w_{\phi}<-1\right)$, indicating that data preferred a late-time expansion rate lower than the one obtained when DE was entirely in the form of a (positive) cosmological constant. We also saw that when moving from the wCDM model to the $c$ CDM model, the central value of $w_{\phi}$, although still phantom, moved upwards towards $w=-1$. The reason is that, as discussed in Section 1, introducing a negative cosmological constant lowers the expansion rate even more drastically than when considering a phantom DE component, lessening the need for the latter and explaining why $w_{\phi}$ moves towards -1 . 
Table 2. Left column: corner plots showing the 1D marginalized and 2D joint posterior distributions for the parameters of the $\Lambda \mathrm{CDM}$ (top panel), $w \mathrm{CDM}$ (middle panel), and $c \mathrm{CDM}$ models (bottom panel), when anchoring BAO measurements to $r_{\text {drag }}$ as measured Planck [5]. Right column: same as the left column, but anchoring BAO measurements to $H_{0}$ as measured by the SHOES program [8].

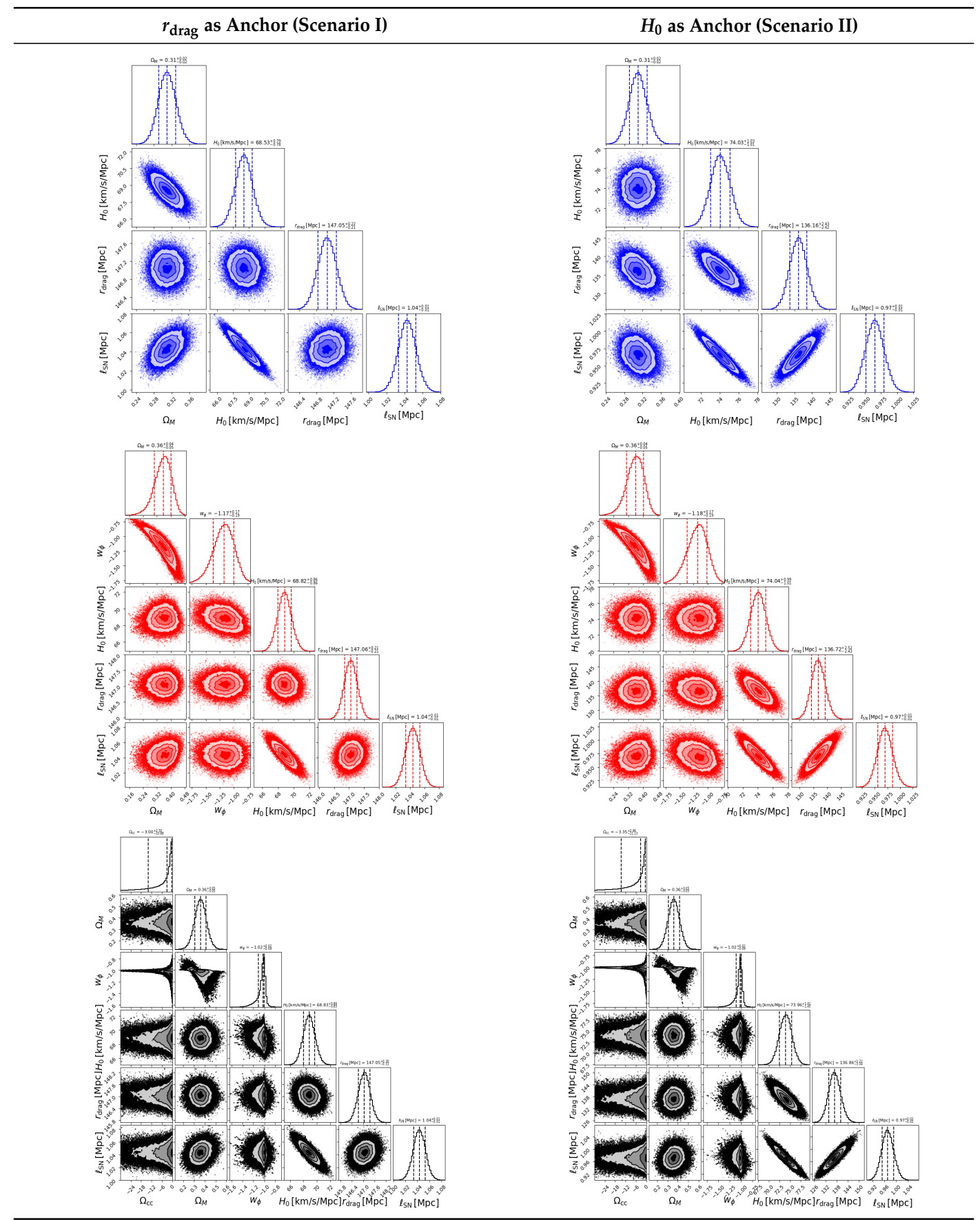


Table 3. Constraints on the parameters of the three models considered in this work, obtained using the two different choices of anchor (specified in the first column). We report $68 \%$ C.L. intervals on all parameters, except for $\Omega_{\mathcal{c}}$, for which we do not have a detection, and hence report the $95 \%$ C.L. lower bound. The last column reports $\triangle \mathrm{AIC}$, defined in Equation (15) and calculated with respect to the $\Lambda \mathrm{CDM}$ model. A positive value of $\triangle \mathrm{AIC}$ indicates a preference for $\Lambda \mathrm{CDM}$.

\begin{tabular}{ccccccccc}
\hline Anchor & Model & $\boldsymbol{\Omega}_{\boldsymbol{c}}$ & $\boldsymbol{w}_{\boldsymbol{\phi}}$ & $\boldsymbol{\Omega}_{\boldsymbol{M}}$ & $\begin{array}{c}\boldsymbol{H}_{\mathbf{0}} \\
\mathbf{k m} / \mathbf{s} / \mathbf{M p c}\end{array}$ & $\begin{array}{c}\boldsymbol{r}_{\boldsymbol{s}} \\
\mathbf{( M p c )}\end{array}$ & $\begin{array}{c}\boldsymbol{\ell}_{\mathbf{S N}} \\
\mathbf{( M p c})\end{array}$ & $\boldsymbol{\Delta} \mathbf{A I C}$ \\
\hline$r_{\text {drag }}$ & $\Lambda \mathrm{CDM}$ & & & $0.31 \pm 0.02$ & $68.53_{-0.78}^{+0.79}$ & $147.05_{-0.21}^{+0.22}$ & $1.04 \pm 0.01$ & 0 \\
$r_{\text {drag }}$ & $w \mathrm{CDM}$ & & $-1.17_{-0.19}^{+0.17}$ & $0.36_{-0.05}^{+0.04}$ & $68.82_{-0.86}^{+0.86}$ & $147.06_{-0.21}^{+0.21}$ & $1.04 \pm 0.01$ & 1.9 \\
$r_{\text {drag }}$ & $c \mathrm{CDM}$ & $>-13.88$ & $-1.02_{-0.09}^{+0.02}$ & $0.36_{-0.05}^{+0.05}$ & $68.83_{-0.85}^{+0.86}$ & $147.05_{-0.31}^{+0.30}$ & $1.04 \pm 0.01$ & 3.6 \\
\hline$H_{0}$ & $\Lambda \mathrm{CDM}$ & & & $0.31 \pm 0.02$ & $74.03_{-1.01}^{+1.03}$ & $136.16_{-2.42}^{+2.43}$ & $0.97 \pm 0.01$ & 0 \\
$H_{0}$ & $w \mathrm{CDM}$ & & $-1.18_{-0.19}^{+0.17}$ & $0.36_{-0.05}^{+0.04}$ & $74.04_{-1.01}^{+0.99}$ & $136.72_{-2.43}^{+2.52}$ & $0.97 \pm 0.01$ & 1.3 \\
$H_{0}$ & $c \mathrm{CDM}$ & $>-14.48$ & $-1.02_{-0.09}^{+0.02}$ & $0.36_{-0.05}^{+0.05}$ & $73.96_{-1.45}^{+1.41}$ & $136.86_{-3.04}^{+3.22}$ & $0.97 \pm 0.02$ & 4.8 \\
\hline
\end{tabular}

It is also worth noting that within the $w \mathrm{CDM}$ and $c \mathrm{CDM}$ model, we infer a higher matter density than within $\Lambda \mathrm{CDM}$ (although with uncertainty about twice as large). The reason is again that a phantom dark energy component (with the addition of a negative cosmological constant) lowers the expansion rate at late times, which can be compensated by increasing the matter density. This result is analogous to the one found in [55], where it was shown that in models with $w>-1$, one infers a lower matter density, because the late-time expansion rate in this case is higher, leaving less room for matter components.

We also notice that when anchoring BAO measurements to $r_{\text {drag }}$ as measured by Planck, we recovered a value of $H_{0}$, which is essentially $H_{0} \approx 68.5 \mathrm{~km} \mathrm{~s}^{-1} \mathrm{Mpc}^{-1}$ for all three models, which indicates that the $c \mathrm{CDM}$ model was not able to alleviate the $H_{0}$ tension. Finally, we note that anchoring BAO measurements to $H_{0}$ as measured by SHOES, we inferred a value for $r_{\text {drag }}$ that was about $8 \%$ lower than that obtained when anchoring to $r_{\mathrm{drag}}$ as measured by Planck, in full agreement with earlier results $[51,108,296]$.

One further interesting point worth noting from Table 3 is that the values we inferred for certain parameters (such as $\Omega_{M}, H_{0}, r_{s}$, and $l_{S N}$ ) were essentially the same across different models (particularly for the $w \mathrm{CDM}$ and $c \mathrm{CDM}$ models) and for different choices of anchors. One particularly striking case, for example, is $\Omega_{M}$, for which we basically inferred $\Omega_{M}=0.36 \pm 0.05$ for both the $w C D M$ and $c$ CDM models, regardless of the choice of anchor. On the other hand, for the $\Lambda$ CDM model, we inferred $\Omega_{M}=0.31 \pm 0.02$. This is consistent with the fact that we did not have a detection of a non-zero negative cosmological constant, and therefore, from the point of view of the data we used, there was not much difference between the $w \mathrm{CDM}$ and $c \mathrm{CDM}$ models. On the other hand, parameters such as $\Omega_{M}$ were extremely well determined by BAO data, independent of the choice of anchor. Similar considerations hold for the other parameters whose values inferred within the $w \mathrm{CDM}$ and $c \mathrm{CDM}$ models were very similar to one another regardless of the choice of anchor.

We now compare the three models using the Akaike information criterion introduced in Section 3 , beginning with the case when BAO measurements are anchored to $r_{\text {drag }}$. In this case, we found minimal improvements in the best-fit $\chi^{2}$ when moving away from the $\Lambda$ CDM model. In particular, we found $\Delta \chi^{2}=-0.1$ and $\Delta \chi^{2}=-0.4$ for the $w C D M$ and $c$ CDM models, respectively. According to Equation (15), these values correspond to $\triangle \mathrm{AIC}=1.9$ and $\triangle \mathrm{AIC}=3.6$ for the $w \mathrm{CDM}$ and $c \mathrm{CDM}$ models, respectively. In both cases $\triangle \mathrm{AIC}>0$, indicating that the tiny improvement in fit did not warrant the addition of extra parameters (one extra parameter and two extra parameters for $w \mathrm{CDM}$ and $c \mathrm{CDM}$, respectively) and, therefore, that the baseline $\Lambda \mathrm{CDM}$ model was preferred from a statistical point of view. In fact, we found that the relative likelihoods of the $w \mathrm{CDM}$ and $c \mathrm{CDM}$ models over $\Lambda \mathrm{CDM}$ were $\approx 0.38$ and $\approx 0.16$, respectively. 
We found completely analogous, if not less optimistic results, when anchoring BAO measurements to $H_{0}$. In this case, we found $\Delta \chi^{2}=-0.7$ and $\Delta \chi^{2}=+0.8$ for the $w C D M$ and $c$ CDM models, respectively. We saw that within the $c \mathrm{CDM}$ model, the quality of the fit had actually worsened. According to Equation (15), these values corresponded to $\triangle \mathrm{AIC}=1.3$ and $\triangle \mathrm{AIC}=4.8$ for the $w \mathrm{CDM}$ and $c$ CDM models, respectively. Again, in both cases, $\triangle \mathrm{AIC}>0$, with relative likelihoods for the $w \mathrm{CDM}$ and $c \mathrm{CDM}$ models over the $\Lambda \mathrm{CDM}$ model being $\approx 0.52$ and $\approx 0.09$, respectively.

\section{Conclusions}

In this work, we revisited the possibility that the dark energy sector might feature two components: a negative cosmological constant and a component with positive energy density (such as a quintessence field, but with the possibility that is equation of state might be phantom) on top. While such a model (which we referred to as $c \mathrm{CDM}$ model) is certainly a toy model, it can be seen as a proxy for more realistic string-inspired models [176-180], where it is rather natural to have an AdS background with on top a large number of light bosons corresponding to moduli determining the size and shape of the extra dimensions. We tested our toy $c \mathrm{CDM}$ model against low-redshift distance measurements from a collection of recent BAO surveys and the Pantheon SNeIa catalogue. To interpret our BAO measurements, we required either an anchor either at high or low redshift. We experimented with two different choices of anchor: a high-redshift anchor based on the sound horizon at the drag epoch $r_{\text {drag }}$ as determined by the Planck collaboration [5] or a low-redshift anchor based on the local distance ladder measurement of the Hubble constant $H_{0}$ measured by the SHOES program [8].

Our results are summarized in Tables 2 and 3. We found no evidence for a negative cosmological constant, but only obtained the very loose lower bound $\Omega_{c} \gtrsim-14$. Moreover, we found a mild preference for a phantom equation of state $\left(w_{\phi}<-1\right)$ for the dark energy component on top of the negative cosmological constant, in agreement with recent works.

We also compared the three models considered in this work: the baseline $\Lambda$ CDM model, the one-parameter $w \mathrm{CDM}$ extension where we allowed the DE EoS $w_{\phi}$ to vary freely, and finally, the $c \mathrm{CDM}$ model with a negative cosmological constant in addition. We found that for most choices of anchor/model, the increased model complexity only marginally improved the quality of the fit (which actually deteriorated when considering the $c \mathrm{CDM}$ model and anchoring BAO measurements to $H_{0}$ ). When accounting for the increased model complexity by comparing the three models using the Akaike information criterion, we found that the baseline $\Lambda \mathrm{CDM}$ model was always statistically favoured over the extensions we considered.

The results of our work were less optimistic than earlier results in, e.g., [93,164,165], which found indications for a negative dark energy density at $z \approx 2$. There were nonetheless some fundamental differences between the approaches of $[93,164,165]$ and ours. We have not attempted to non-parametrically reconstruct the dark energy density, but rather focused on a string-inspired toy model, which we then fitted to the data. Moreover, on top of the negative cosmological constant, we considered a dark energy component with a constant equation of state, although an obvious extension would be to consider a time-varying equation of state (which is very natural for a quintessence model). In any case, our results suggest that a negative cosmological constant is certainly consistent with data. Should the trend that sees low-redshift data favouring a lower expansion rate continue (for instance in light of the persisting $H_{0}$ tension), our scenario featuring a negative cosmological constant with a quintessence component on top might be worth reconsidering. It is worth reminding once more that, while being a toy model, our $c \mathrm{CDM}$ model nonetheless enjoyed a fundamental string-inspired motivation.

Finally, there are several avenues by which we can extend and improve the current work. From the observational side, an analysis performing a full fit to CMB data from the Planck satellite, following the upcoming public release of the 2019 legacy likelihood, would be valuable. It would also be worth allowing for more freedom in the dark energy sector, for instance by considering a time-varying dark energy component on top of the negative cosmological constant (in this work, we restricted ourselves 
to the case where the equation of state was a constant). Finally, it would also be interesting to consider forecasts for how our constraints could improve when data from future CMB experiments become available (such as Simons Observatory [297,298] or CMB-S4 [299]) or gravitational wave standard sirens will improve constraints on the late-time expansion rate (e.g., along the lines of [300]). On the theory side, it would be worth further refining our toy model by more carefully modelling the negative cosmological constant and quintessence component emerging from realistic string constructions. We plan to report on these and other issues in upcoming work.

Author Contributions: Conceptualization, L.V., S.V., and U.D.; methodology, L.V. and S.V.; software, L.V. and S.V.; formal analysis, L.V.; writing, original draft preparation, L.V.; writing, review and editing, L.V., S.V., and U.D.; supervision, U.D.

Funding: L.V. and S.V. are funded by the Vetenskapsrådet (Swedish Research Council) through Contract No. 638-2013-8993 and the Oskar Klein Centre for Cosmoparticle Physics. S.V. is additionally funded by the Isaac Newton Trust and the Kavli Foundation through a Newton-Kavli fellowship. U.D. is funded by the Vetenskapsrådet (Swedish Research Council) under Contract 2015-04814.

Acknowledgments: We thank Massimiliano Lattanzi for useful suggestions that significantly improved this work. L.V. acknowledges the kind hospitality of the INFN Laboratori Nazionali di Frascati and the Leinweber Center for Theoretical Physics where part of this work was carried out.

Conflicts of Interest: The authors declare no conflict of interest. The funders had no role in the design of the study; in the collection, analyses, or interpretation of data; in the writing of the manuscript; nor in the decision to publish the results.

\section{Abbreviations}

The following abbreviations are used in this manuscript:

$\begin{array}{ll}\text { AdS } & \text { anti-de Sitter } \\ \text { AIC } & \text { Akaike Information Criterion } \\ \text { BAO } & \text { Baryon Acoustic Oscillations } \\ \text { BOSS } & \text { Baryon Oscillation Spectroscopic Survey } \\ \text { CMB } & \text { Cosmic Microwave Background } \\ \text { DE } & \text { Dark Energy } \\ \text { DR12 } & \text { Data Release 12 } \\ \text { dS } & \text { de Sitter } \\ \text { eBOSS } & \text { Extended Baryon Oscillation Spectroscopic Survey } \\ \text { EoS } & \text { Equation of State } \\ \text { HST } & \text { Hubble Space Telescope } \\ \text { MCMC } & \text { Markov Chain Monte Carlo } \\ \text { MGS } & \text { Main Galaxy Sample } \\ \text { SDSS } & \text { Sloan Digital Sky Survey } \\ \text { SH0ES } & \text { Supernovae and H0 for the Dark Energy Equation of State } \\ \text { SNeIa } & \text { Type Ia Supernovae } \\ \text { 6dFGS } & \text { Six-degree Field Galaxy Survey }\end{array}$

\section{References}

1. Riess, A.G.; Filippenko, A.V.; Challis, P.; Clocchiatti, A.; Diercks, A.; Garnavich, P.M.; Gilliland, R.L.; Hogan, C.J.; Jha, S.; Kirshner, R.P.; et al. Observational evidence from supernovae for an accelerating universe and a cosmological constant. Astron. J. 1998, 116, 1009-1038. [CrossRef]

2. Perlmutter, S.; Aldering, G.; Goldhaber, G.; Knop, R.A.; Nugent, P.; Castro, P.G.; Deustua1, S.; Fabbro, S.; Goobar, A.; Groom, D.E.; et al. Measurements of Omega and Lambda from 42 high redshift supernovae. Astrophys. J. 1999, 517, 565-586. [CrossRef]

3. Alam, S.; Ata, M.; Bailey, S.; Beutler, F.; Bizyaev, D.; Blazek, J.A.; Bolton, A.S.; Brownstein, J.R.; Burden, A.; Chuang, C.; et al. The clustering of galaxies in the completed SDSS-III Baryon Oscillation Spectroscopic Survey: Cosmological analysis of the DR12 galaxy sample. Mon. Not. R. Astron. Soc. 2017, 470, 2617-2652. [CrossRef] 
4. Dark Energy Survey Collaboration. Dark Energy Survey Year 1 results: Cosmological constraints from cosmic shear. Phys. Rev. D 2018, 98, 043528. [CrossRef]

5. Aghanim, N.; Akrami, Y.; Ashdown, M.; Aumont, J.; Baccigalupi, C.; Ballardini, M.; Banday, A.J.; Barreiro, R.B.; Bartolo, N.; Basak, S.; et al. Planck 2018 results. VI. Cosmological parameters. arXiv 2018, arXiv:1807.06209.

6. Freedman, W.L. Cosmology at a Crossroads. Nat. Astron. 2017, 1, 0121. [CrossRef]

7. Di Valentino, E. Crack in the cosmological paradigm. Nat. Astron. 2017, 1, 569-570. [CrossRef]

8. Riess, A.G.; Casertano, S.; Yuan, W.; Macri, L.M.; Scolnic, D. Large Magellanic Cloud Cepheid Standards Provide a $1 \%$ Foundation for the Determination of the Hubble Constant and Stronger Evidence for Physics Beyond LambdaCDM. Astrophys. J. 2019, 876, 85. [CrossRef]

9. de Blok, W.J.G. The Core-Cusp Problem. Adv. Astron. 2010, 2010, 789293. [CrossRef]

10. Klypin, A.A.; Kravtsov, A.V.; Valenzuela, O.; Prada, F. Where are the missing Galactic satellites? Astrophys. J. 1999, 522, 82-92. [CrossRef]

11. Simon, J.D.; Geha, M. The Kinematics of the Ultra-Faint Milky Way Satellites: Solving the Missing Satellite Problem. Astrophys. J. 2007, 670, 313-331. [CrossRef]

12. Kim, S.Y.; Peter, A.H.G.; Hargis, J.R. Missing Satellites Problem: Completeness Corrections to the Number of Satellite Galaxies in the Milky Way are Consistent with Cold Dark Matter Predictions. Phys. Rev. Lett. 2018, 121, 211302. [CrossRef] [PubMed]

13. Boylan-Kolchin, M.; Bullock, J.S.; Kaplinghat, M. Too big to fail? The puzzling darkness of massive Milky Way subhaloes. Mon. Not. R. Astron. Soc. 2011, 415, L40. [CrossRef]

14. Weinberg, D.H.; Bullock, J.S.; Governato, F.; Kuzio de Naray, R.; Peter, A.H.G. Cold dark matter: Controversies on small scales. Proc. Nat. Acad. Sci. USA 2015, 112, 12249-12255. [CrossRef] [PubMed]

15. Del Popolo, A.; Le Delliou, M. Small scale problems of the $\Lambda$ CDM model: A short review. Galaxies 2017, 5, 17. [CrossRef]

16. Bullock, J.S.; Boylan-Kolchin, M. Small-Scale Challenges to the $\Lambda$ CDM Paradigm. Ann. Rev. Astron. Astrophys. 2017, 55, 343-387. [CrossRef]

17. Spergel, D.N.; Steinhardt, P.J. Observational evidence for selfinteracting cold dark matter. Phys. Rev. Lett. 2000, 84, 3760-3763. [CrossRef] [PubMed]

18. Strigari, L.E.; Bullock, J.S.; Kaplinghat, M.; Diemand, J.; Kuhlen, M.; Madau, P. Redefining the Missing Satellites Problem. Astrophys. J. 2007, 669, 676-683. [CrossRef]

19. van den Aarssen, L.G.; Bringmann, T.; Pfrommer, C. Is dark matter with long-range interactions a solution to all small-scale problems of $\Lambda$ CDM cosmology? Phys. Rev. Lett. 2012, 109, 231301. [CrossRef]

20. Tulin, S.; Yu, H.B.; Zurek, K.M. Beyond Collisionless Dark Matter: Particle Physics Dynamics for Dark Matter Halo Structure. Phys. Rev. D 2013, 87, 115007. [CrossRef]

21. Foot, R.; Vagnozzi, S. Dissipative hidden sector dark matter. Phys. Rev. D 2015, 91, 023512. [CrossRef]

22. Foot, R.; Vagnozzi, S. Diurnal modulation signal from dissipative hidden sector dark matter. Phys. Lett. B 2015, 748, 61-66. [CrossRef]

23. Kaplinghat, M.; Tulin, S.; Yu, H.B. Dark Matter Halos as Particle Colliders: Unified Solution to Small-Scale Structure Puzzles from Dwarfs to Clusters. Phys. Rev. Lett. 2016, 116, 041302. [CrossRef] [PubMed]

24. Foot, R.; Vagnozzi, S. Solving the small-scale structure puzzles with dissipative dark matter. JCAP 2016, 1607, 013. [CrossRef]

25. Binder, T.; Covi, L.; Kamada, A.; Murayama, H.; Takahashi, T.; Yoshida, N. Matter Power Spectrum in Hidden Neutrino Interacting Dark Matter Models: A Closer Look at the Collision Term. JCAP 2016, 1611, 043. [CrossRef]

26. Tang, Y. Interacting Scalar Radiation and Dark Matter in Cosmology. Phys. Lett. B 2016, 757, 387-392, [CrossRef]

27. Bringmann, T.; Ihle, H.T.; Kersten, J.; Walia, P. Suppressing structure formation at dwarf galaxy scales and below: Late kinetic decoupling as a compelling alternative to warm dark matter. Phys. Rev. D 2016, 94, 103529. [CrossRef]

28. Du, X.; Behrens, C.; Niemeyer, J.C. Substructure of fuzzy dark matter haloes. Mon. Not. R. Astron. Soc. 2017, 465, 941-951. [CrossRef]

29. Tulin, S.; Yu, H.B. Dark Matter Self-interactions and Small Scale Structure. Phys. Rep. 2018, 730, 1-57. [CrossRef] 
30. Deng, H.; Hertzberg, M.P.; Namjoo, M.H.; Masoumi, A. Can Light Dark Matter Solve the Core-Cusp Problem? Phys. Rev. D 2018, 98, 023513. [CrossRef]

31. Robles, V.H.; Bullock, J.S.; Boylan-Kolchin, M. Scalar Field Dark Matter: Helping or Hurting Small-Scale Problems in Cosmology? Mon. Not. R. Astron. Soc. 2019, 483, 289-298. [CrossRef]

32. Riess, A.G.; Macri, L.; Casertano, S.; Sosey, M.; Lampeitl, H.; Ferguson, H.C.; Filippenko, A.V.; Jha, S.W.; Li, W.; Chornock, R.; et al. A Redetermination of the Hubble Constant with the Hubble Space Telescope from a Differential Distance Ladder. Astrophys. J. 2009, 699, 539-563. [CrossRef]

33. Riess, A.G.; Macri, L.; Casertano, S.; Lampeitl, H.; Ferguson, H.C.; Filippenko, A.V.; Jha, S.W.; Li, W.; Chornock, R. A 3\% Solution: Determination of the Hubble Constant with the Hubble Space Telescope and Wide Field Camera 3. Astrophys. J. 2011, 730, 119. [CrossRef]

34. Riess, A.G.; Macri, L.M.; Hoffmann, S.L.; Scolnic, D.; Casertano, S.; Filippenko, A.V.; Tucker, B.E.; Reid, M.J.; Jones, D.O.; Silverman, J.M.; et al. A $2.4 \%$ Determination of the Local Value of the Hubble Constant. Astrophys. J. 2016, 826, 56. [CrossRef]

35. Riess, A.G.; Casertano, S.; Yuan, W.; Macri, L.; Anderson, J.; MacKenty, J.W.; Bowers, J.B.; Clubb, K.I.; Filippenko, A.V.; Jones, D.O.; et al. New Parallaxes of Galactic Cepheids from Spatially Scanning the Hubble Space Telescope: Implications for the Hubble Constant. Astrophys. J. 2018, 855, 136. [CrossRef]

36. Efstathiou, G. H0 Revisited. Mon. Not. R. Astron. Soc. 2014, 440, 1138-1152. [CrossRef]

37. Cardona, W.; Kunz, M.; Pettorino, V. Determining $H_{0}$ with Bayesian hyper-parameters. JCAP 2017, $1703,056$. [CrossRef]

38. Zhang, B.R.; Childress, M.J.; Davis, T.M.; Karpenka, N.V.; Lidman, C.; Schmidt, B.P.; Smith, M. A blinded determination of $H_{0}$ from low-redshift Type Ia supernovae, calibrated by Cepheid variables. Mon. Not. R. Astron. Soc. 2017, 471, 2254-2285. [CrossRef]

39. Feeney, S.M.; Mortlock, D.J.; Dalmasso, N. Clarifying the Hubble constant tension with a Bayesian hierarchical model of the local distance ladder. Mon. Not. R. Astron. Soc. 2018, 476, 3861-3882. [CrossRef]

40. Dhawan, S.; Jha, S.W.; Leibundgut, B. Measuring the Hubble constant with Type Ia supernovae as near-infrared standard candles. Astron. Astrophys. 2018, 609, A72. [CrossRef]

41. Follin, B.; Knox, L. Insensitivity of the distance ladder Hubble constant determination to Cepheid calibration modelling choices. Mon. Not. R. Astron. Soc. 2018, 477, 4534-4542. [CrossRef]

42. Gómez-Valent, A.; Amendola, L. $H_{0}$ from cosmic chronometers and Type Ia supernovae, with Gaussian Processes and the novel Weighted Polynomial Regression method. JCAP 2018, 1804, 051. [CrossRef]

43. Burns, C.R.; Parent, E.; Phillips, M.M.; Stritzinger, M.; Krisciunas, K.; Suntzeff, N.B.; Hsiao, E.Y.; Contreras, C.; Anais, J.; Boldt, L.; et al. The Carnegie Supernova Project: Absolute Calibration and the Hubble Constant. Astrophys. J. 2018, 869, 56. [CrossRef]

44. Collett, T.; Montanari, F.; Rasanen, S. Model-independent determination of $H_{0}$ and $\Omega_{K 0}$ from strong lensing and type Ia supernovae. arXiv 2019, arXiv:1905.09781.

45. Camarena, D.; Marra, V. Cosmology-independent local determination of $H_{0}$ in strong tension with CMB. arXiv 2019, arXiv:1906.11814.

46. Freedman, W.L.; Madore, B.F.; Hatt, D.; Hoyt, T.J.; Jang, I.S.; Beaton, R.L.; Burns, C.R.; Lee, M.G.; Monson, A.J.; Neeley, J.R.; et al. The Carnegie-Chicago Hubble Program. VIII. An Independent Determination of the Hubble Constant Based on the Tip of the Red Giant Branch. arXiv 2019, arXiv:1907.05922.

47. Bonvin, V.; Courbin, F.; Suyu, S.H.; Marshall, P.J.; Rusu, C.E.; Sluse, D.; Tewes, M.; Wong, K.C.; Collett, T.; Fassnacht, C.D.; et al. HOLiCOW-V. New COSMOGRAIL time delays of HE 0435-1223: $H_{0}$ to 3.8 per cent precision from strong lensing in a flat $\Lambda$ CDM model. Mon. Not. R. Astron. Soc. 2017, 465, 4914-4930. [CrossRef]

48. Birrer, S.; Treu, T.; Rusu, C.E.; Bonvin, V.; Fassnacht, C.D.; Chan, J.H.H. H0LiCOW-IX. Cosmographic analysis of the doubly imaged quasar SDSS $1206+4332$ and a new measurement of the Hubble constant. Mon. Not. R. Astron. Soc. 2019, 484, 4726. [CrossRef]

49. Wong, K.C.; Suyu, S.H.; Chen, G.C.F.; Rusu, C.E.; Millon, M.; Sluse, D. H0LiCOW XIII. A 2.4\% measurement of $H_{0}$ from lensed quasars: $5.3 \sigma$ tension between early and late-Universe probes. arXiv 2019, arXiv:1907.04869.

50. Lombriser, L. Consistency of the local Hubble constant with the cosmic microwave background. arXiv 2019, arXiv:1906.12347.

51. Bernal, J.L.; Verde, L.; Riess, A.G. The trouble with $H_{0}$. JCAP 2016, 1610, 019. [CrossRef] 
52. Di Valentino, E.; Melchiorri, A.; Silk, J. Reconciling Planck with the local value of $H_{0}$ in extended parameter space. Phys. Lett. B 2016, 761, 242-246. [CrossRef]

53. Mörtsell, E.; Dhawan, S. Does the Hubble constant tension call for new physics? JCAP 2018, $1809,025$. [CrossRef]

54. Guo, R.Y.; Zhang, J.F.; Zhang, X. Can the $H_{0}$ tension be resolved in extensions to $\Lambda$ CDM cosmology? JCAP 2019, 1902, 054. [CrossRef]

55. Vagnozzi, S.; Dhawan, S.; Gerbino, M.; Freese, K.; Goobar, A.; Mena, O. Constraints on the sum of the neutrino masses in dynamical dark energy models with $w(z) \geq-1$ are tighter than those obtained in $\Lambda$ CDM. Phys. Rev. D 2018, 98, 083501. [CrossRef]

56. Di Valentino, E.; Melchiorri, A.; Silk, J. Cosmological constraints in extended parameter space from the Planck 2018 Legacy release. arXiv 2019, arXiv:1908.01391.

57. Di Valentino, E.; Ferreira, R.Z.; Visinelli, L.; Danielsson, U. Late time transitions in the quintessence field and the $H_{0}$ tension. arXiv 2019, arXiv:1906.11255.

58. Barreira, A.; Li, B.; Baugh, C.; Pascoli, S. Modified gravity with massive neutrinos as a testable alternative cosmological model. Phys. Rev. D 2014, 90, 023528. [CrossRef]

59. Murgia, R.; Gariazzo, S.; Fornengo, N. Constraints on the Coupling between Dark Energy and Dark Matter from CMB data. JCAP 2016, 1604, 014. [CrossRef]

60. Huang, Q.G.; Wang, K. How the dark energy can reconcile Planck with local determination of the Hubble constant. Eur. Phys. J. C 2016, 76, 506. [CrossRef]

61. Tram, T.; Vallance, R.; Vennin, V. Inflation Model Selection meets Dark Radiation. JCAP 2017, $1701,046$. [CrossRef]

62. Ko, P.; Tang, Y. Light dark photon and fermionic dark radiation for the Hubble constant and the structure formation. Phys. Lett. B 2016, 762, 462-466. [CrossRef]

63. Karwal, T.; Kamionkowski, M. Dark energy at early times, the Hubble parameter, and the string axiverse. Phys. Rev. D 2016, 94, 103523. [CrossRef]

64. Kumar, S.; Nunes, R.C. Probing the interaction between dark matter and dark energy in the presence of massive neutrinos. Phys. Rev. D 2016, 94, 123511. [CrossRef]

65. Xia, D.M.; Wang, S. Constraining interacting dark energy models with latest cosmological observations. Mon. Not. R. Astron. Soc. 2016, 463, 952-956. [CrossRef]

66. Chacko, Z.; Cui, Y.; Hong, S.; Okui, T.; Tsai, Y. Partially Acoustic Dark Matter, Interacting Dark Radiation, and Large Scale Structure. JHEP 2016, 12, 108. [CrossRef]

67. Zhao, G.B.; Raveri, M.; Pogosian, L.; Wang, Y.; Crittenden, R.G.; H.; ley, W.J. Dynamical dark energy in light of the latest observations. Nat. Astron. 2017, 1, 627-632. [CrossRef]

68. Kumar, S.; Nunes, R.C. Echo of interactions in the dark sector. Phys. Rev. D 2017, 96, 103511. [CrossRef]

69. Agrawal, P.; Cyr-Racine, F.Y.; Randall, L.; Scholtz, J. Dark Catalysis. JCAP 2017, 1708, 021. [CrossRef]

70. Benetti, M.; Graef, L.L.; Alcaniz, J.S. Do joint CMB and HST data support a scale invariant spectrum? JCAP 2017, 1704, 003. [CrossRef]

71. Feng, L.; Zhang, J.F.; Zhang, X. A search for sterile neutrinos with the latest cosmological observations. Eur. Phys. J. C 2017, 77, 418. [CrossRef]

72. Zhao, M.M.; He, D.Z.; Zhang, J.F.; Zhang, X. Search for sterile neutrinos in holographic dark energy cosmology: Reconciling Planck observation with the local measurement of the Hubble constant. Phys. Rev. D 2017, 96, 043520. [CrossRef]

73. Di Valentino, E.; Melchiorri, A.; Linder, E.V.; Silk, J. Constraining Dark Energy Dynamics in Extended Parameter Space. Phys. Rev. D 2017, 96, 023523. [CrossRef]

74. Gariazzo, S.; Escudero, M.; Diamanti, R.; Mena, O. Cosmological searches for a noncold dark matter component. Phys. Rev. D 2017, 96, 043501. [CrossRef]

75. Dirian, Y. Changing the Bayesian prior: Absolute neutrino mass constraints in nonlocal gravity. Phys. Rev. D 2017, 96, 083513. [CrossRef]

76. Di Valentino, E.; Melchiorri, A.; Mena, O. Can interacting dark energy solve the $H_{0}$ tension? Phys. Rev. D 2017, 96, 043503. [CrossRef]

77. Solà, J.; Gómez-Valent, A.; de Cruz Pérez, J. The $H_{0}$ tension in light of vacuum dynamics in the Universe. Phys. Lett. B 2017, 774, 317-324. [CrossRef] 
78. Feng, L.; Zhang, J.F.; Zhang, X. Searching for sterile neutrinos in dynamical dark energy cosmologies. Sci. China Phys. Mech. Astron. 2018, 61, 050411. [CrossRef]

79. Renk, J.; Zumalacárregui, M.; Montanari, F.; Barreira, A. Galileon gravity in light of ISW, CMB, BAO and $\mathrm{H}_{0}$ data. JCAP 2017, 1710, 020. [CrossRef]

80. Yang, W.; Pan, S.; Paliathanasis, A. Latest astronomical constraints on some non-linear parametric dark energy models. Mon. Not. R. Astron. Soc. 2018, 475, 2605-2613. [CrossRef]

81. Buen-Abad, M.A.; Schmaltz, M.; Lesgourgues, J.; Brinckmann, T. Interacting Dark Sector and Precision Cosmology. JCAP 2018, 1801, 008. [CrossRef]

82. Yang, W.; Pan, S.; Mota, D.F. Novel approach toward the large-scale stable interacting dark-energy models and their astronomical bounds. Phys. Rev. D 2017, 96, 123508. [CrossRef]

83. Raveri, M.; Hu, W.; Hoffman, T.; Wang, L.T. Partially Acoustic Dark Matter Cosmology and Cosmological Constraints. Phys. Rev. D 2017, 96, 103501. [CrossRef]

84. Di Valentino, E.; Linder, E.V.; Melchiorri, A. Vacuum phase transition solves the $H_{0}$ tension. Phys. Rev. D 2018, 97, 043528. [CrossRef]

85. Di Valentino, E.; Bøehm, C.; Hivon, E.; Bouchet, F.R. Reducing the $H_{0}$ and $\sigma_{8}$ tensions with Dark Matter-neutrino interactions. Phys. Rev. D 2018, 97, 043513. [CrossRef]

86. Khosravi, N.; Baghram, S.; Afshordi, N.; Altamirano, N. $H_{0}$ tension as a hint for a transition in gravitational theory. Phys. Rev. D 2019, 99, 103526. [CrossRef]

87. Santos, M.A.; Benetti, M.; Alcaniz, J.; Brito, F.A.; Silva, R. CMB constraints on $\beta$-exponential inflationary models. JCAP 2018, 1803, 023. [CrossRef]

88. Peirone, S.; Frusciante, N.; Hu, B.; Raveri, M.; Silvestri, A. Do current cosmological observations rule out all Covariant Galileons? Phys. Rev. D 2018, 97, 063518. [CrossRef]

89. Benetti, M.; Graef, L.L.; Alcaniz, J.S. The $H_{0}$ and $\sigma_{8}$ tensions and the scale invariant spectrum. JCAP 2018, 1807, 066. [CrossRef]

90. Feng, L.; Zhang, J.F.; Zhang, X. Search for sterile neutrinos in a universe of vacuum energy interacting with cold dark matter. Phys. Dark Univ. 2019, 23, 100261. [CrossRef]

91. Belgacem, E.; Dirian, Y.; Foffa, S.; Maggiore, M. Nonlocal gravity. Conceptual aspects and cosmological predictions. JCAP 2018, 1803, 002. [CrossRef]

92. Nunes, R.C. Structure formation in $f(T)$ gravity and a solution for $H_{0}$ tension. JCAP 2018, $1805,052$. [CrossRef]

93. Poulin, V.; Boddy, K.K.; Bird, S.; Kamionkowski, M. Implications of an extended dark energy cosmology with massive neutrinos for cosmological tensions. Phys. Rev. D 2018, 97, 123504. [CrossRef]

94. Kumar, S.; Nunes, R.C.; Yadav, S.K. Cosmological bounds on dark matter-photon coupling. Phys. Rev. D 2018, 98, 043521. [CrossRef]

95. Yang, W.; Pan, S.; Xu, L.; Mota, D.F. Effects of anisotropic stress in interacting dark matter-Dark energy scenarios. Mon. Not. R. Astron. Soc. 2019, 482, 1858-1871. [CrossRef]

96. Yang, W.; Pan, S.; Di Valentino, E.; Nunes, R.C.; Vagnozzi, S.; Mota, D.F. Tale of stable interacting dark energy, observational signatures, and the $H_{0}$ tension. JCAP 2018, 1809, 019. [CrossRef]

97. Yang, W.; Pan, S.; Herrera, R.; Chakraborty, S. Large-scale (in) stability analysis of an exactly solved coupled dark-energy model. Phys. Rev. D 2018, 98, 043517. [CrossRef]

98. Banihashemi, A.; Khosravi, N.; Shirazi, A.H. Ups and Downs in Dark Energy: Phase transition in dark sector as a proposal to lessen cosmological tensions. arXiv 2018, arXiv:1808.02472.

99. D’Eramo, F.; Ferreira, R.Z.; Notari, A.; Bernal, J.L. Hot Axions and the $H_{0}$ tension. JCAP 2018, $1811,014$. [CrossRef]

100. de Martino, I. Decaying dark energy in light of the latest cosmological dataset. Symmetry 2018, 10, 372. [CrossRef]

101. Graef, L.L.; Benetti, M.; Alcaniz, J.S. Primordial gravitational waves and the H0-tension problem. Phys. Rev. D 2019, 99, 043519. [CrossRef]

102. Yang, W.; Mukherjee, A.; Di Valentino, E.; Pan, S. Interacting dark energy with time varying equation of state and the $H_{0}$ tension. Phys. Rev. D 2018, 98, 123527. [CrossRef]

103. Benevento, G.; Raveri, M.; Lazanu, A.; Bartolo, N.; Liguori, M.; Brax, P.; Valageas, P. K-mouflage Imprints on Cosmological Observables and Data Constraints. JCAP 2019, 1905, 027. [CrossRef] 
104. Lin, M.X.; Raveri, M.; Hu, W. Phenomenology of Modified Gravity at Recombination. Phys. Rev. D 2019, 99, 043514. [CrossRef]

105. Yang, W.; Pan, S.; Di Valentino, E.; Saridakis, E.N.; Chakraborty, S. Observational constraints on one-parameter dynamical dark-energy parametrizations and the $H_{0}$ tension. Phys. Rev. D 2019, 99, 043543. [CrossRef]

106. Yang, W.; Shahalam, M.; Pal, B.; Pan, S.; Wang, A. Cosmological constraints on quintessence scalar field models against the astronomical observations. arXiv 2018, arXiv:1810.08586.

107. Banihashemi, A.; Khosravi, N.; Shirazi, A.H. Ginzburg-Landau Theory of Dark Energy: A Framework to Study Both Temporal and Spatial Cosmological Tensions Simultaneously. Phys. Rev. D 2019, 99, 083509. [CrossRef]

108. Aylor, K.; Joy, M.; Knox, L.; Millea, M.; Raghunathan, S.; Wu, W.L.K. Sounds Discordant: Classical Distance Ladder \& $\Lambda$ CDM -based Determinations of the Cosmological Sound Horizon. Astrophys. J. 2019, 874, 4. [CrossRef]

109. Chiang, C.T.; Slosar, A. Inferences of $H_{0}$ in presence of a non-standard recombination. arXiv 2018, arXiv:1811.03624.

110. Poulin, V.; Smith, T.L.; Karwal, T.; Kamionkowski, M. Early Dark Energy Can Resolve The Hubble Tension. arXiv 2018, arXiv:1811.04083.

111. Carneiro, S.; de Holanda, P.C.; Pigozzo, C.; Sobreira, F. Is the $H_{0}$ tension suggesting a 4th neutrino's generation? Phys. Rev. D 2019, 100, 023505. [CrossRef]

112. Lazkoz, R.; Ortiz-Baños, M.; Salzano, V. The Umami Chaplygin Model. Phys. Dark Univ. 2019, $24,100279$. [CrossRef]

113. Kreisch, C.D.; Cyr-Racine, F.Y.; Doré, O. The Neutrino Puzzle: Anomalies, Interactions, and Cosmological Tensions. arXiv 2019, arXiv:1902.00534.

114. Pandey, K.L.; Karwal, T.; Das, S. Alleviating the $H_{0}$ and $\sigma_{8}$ anomalies with a decaying dark matter model. arXiv 2019, arXiv:1902.10636.

115. Kumar, S.; Nunes, R.C.; Yadav, S.K. Dark sector interaction: A remedy of the tensions between CMB and LSS data. arXiv 2019, arXiv:1903.04865.

116. Vattis, K.; Koushiappas, S.M.; Loeb, A. Late universe decaying dark matter can relieve the $H_{0}$ tension. arXiv 2019, arXiv:1903.06220.

117. Pan, S.; Yang, W.; Singha, C.; Saridakis, E.N. Observational constraints on sign-changeable interaction models and alleviation of the $H_{0}$ tension. arXiv 2019, arXiv:1903.10969.

118. Colgain, E.O. Recasting $H_{0}$ tension as $\Omega_{m}$ tension at low z. arXiv 2019, arXiv:1903.11743.

119. Agrawal, P.; Cyr-Racine, F.Y.; Pinner, D.; Randall, L. Rock ' $n$ ' Roll Solutions to the Hubble Tension. arXiv 2019, arXiv:1904.01016.

120. Alexander, S.; McDonough, E. Axion-Dilaton Destabilization and the Hubble Tension. arXiv 2019, arXiv:1904.08912.

121. Yang, W.; Pan, S.; Paliathanasis, A.; Ghosh, S.; Wu, Y. Observational constraints of a new unified dark fluid and the $H_{0}$ tension. arXiv 2019, arXiv:1904.10436.

122. Adhikari, S.; Huterer, D. Super-CMB fluctuations can resolve the Hubble tension. arXiv 2019, arXiv:1905.02278.

123. Keeley, R.E.; Joudaki, S.; Kaplinghat, M.; Kirkby, D. Implications of a transition in the dark energy equation of state for the $H_{0}$ and $\sigma_{8}$ tensions. arXiv 2019, arXiv:1905.10198.

124. Peirone, S.; Benevento, G.; Frusciante, N.; Tsujikawa, S. First cosmological constraints and phenomenology of a beyond-Horndeski model. arXiv 2019, arXiv:1905.11364.

125. Lin, M.X.; Benevento, G.; Hu, W.; Raveri, M. Acoustic Dark Energy: Potential Conversion of the Hubble Tension. arXiv 2019, arXiv:1905.12618.

126. Yang, W.; Pan, S.; Di Valentino, E.; Paliathanasis, A.; Lu, J. Challenging bulk viscous unified scenarios with cosmological observations. arXiv 2019, arXiv:1906.04162.

127. Cerdonio, M. The H0 tension conundrum: Did a QCD meV axion emerge? arXiv 2019, arXiv:1906.07080.

128. Agrawal, P.; Obied, G.; Vafa, C. $H_{0}$ Tension, Swampland Conjectures and the Epoch of Fading Dark Matter. arXiv 2019, arXiv:1906.08261.

129. Li, X.; Shafieloo, A. Phenomenologically Emergent Dark Energy and Ruling Out Cosmological Constant. arXiv 2019, arXiv:1906.08275. 
130. Gelmini, G.B.; Kusenko, A.; Takhistov, V. Hints of Sterile Neutrinos in Recent Measurements of the Hubble Parameter. arXiv 2019, arXiv:1906.10136.

131. Yang, W.; Mena, O.; Pan, S.; Di Valentino, E. Dark sectors with dynamical coupling. arXiv 2019, arXiv:1906.11697.

132. Archidiacono, M.; Hooper, D.C.; Murgia, R.; Bohr, S.; Lesgourgues, J.; Viel, M. Constraining Dark Matter-Dark Radiation interactions with CMB, BAO, and Lyman- $\alpha$. arXiv 2019, arXiv:1907.01496.

133. Desmond, H.; Jain, B.; Sakstein, J. A local resolution of the Hubble tension: The impact of screened fifth forces on the cosmic distance ladder. arXiv 2019, arXiv:1907.03778.

134. Yang, W.; Pan, S.; Vagnozzi, S.; Di Valentino, E.; Mota, D.F.; Capozziello, S. Dawn of the dark: Unified dark sectors and the EDGES Cosmic Dawn 21-cm signal. arXiv 2019, arXiv:1907.05344.

135. Nesseris, S.; Sapone, D.; Sypsas, S. Evaporating primordial black holes as varying dark energy. arXiv 2019, arXiv:1907.05608.

136. Baldes, I.; Chowdhury, D.; Tytgat, M.H.G. Forays into the dark side of the swamp. arXiv 2019, arXiv:1907.06663.

137. Pan, S.; Yang, W.; Di Valentino, E.; Saridakis, E.N.; Chakraborty, S. Interacting scenarios with dynamical dark energy: Observational constraints and alleviation of the $H_{0}$ tension. arXiv 2019, arXiv:1907.07540.

138. Vagnozzi, S. New physics in light of the $H_{0}$ tension: An alternative view. arXiv 2019, arXiv:1907.07569.

139. Cai, Y.F.; Khurshudyan, M.; Saridakis, E.N. Model-independent reconstruction of $f(T)$ gravity from Gaussian Processes and alleviation of the $H_{0}$ tension. arXiv 2019, arXiv:1907.10813.

140. Schoneberg, N.; Lesgourgues, J.; Hooper, D.C. The BAO+BBN take on the Hubble tension. arXiv 2019, arXiv:1907.11594.

141. Pan, S.; Yang, W.; Di Valentino, E.; Shafieloo, A.; Chakraborty, S. Reconciling $H_{0}$ tension in a six parameter space? arXiv 2019, arXiv:1907.12551.

142. Caldwell, R.R.; Kamionkowski, M.; Weinberg, N.N. Phantom energy and cosmic doomsday. Phys. Rev. Lett. 2003, 91, 071301. [CrossRef]

143. Nojiri, S.; Odintsov, S.D.; Tsujikawa, S. Properties of singularities in (phantom) dark energy universe. Phys. Rev. D 2005, 71, 063004. [CrossRef]

144. Zhang, X. Heal the world: Avoiding the cosmic doomsday in the holographic dark energy model. Phys. Lett. B 2010, 683, 81-87. [CrossRef]

145. Bamba, K.; Odintsov, S.D.; Sebastiani, L.; Zerbini, S. Finite-time future singularities in modified Gauss-Bonnet and $\mathrm{F}(\mathrm{R}, \mathrm{G})$ gravity and singularity avoidance. Eur. Phys. J. C 2010, 67, 295-310. [CrossRef]

146. Astashenok, A.V.; Nojiri, S.; Odintsov, S.D.; Yurov, A.V. Phantom Cosmology without Big Rip Singularity. Phys. Lett. B 2012, 709, 396-403. [CrossRef]

147. Mörtsell, E. Cosmological histories in bimetric gravity: A graphical approach. JCAP 2017, $1702,051$. [CrossRef]

148. Damour, T.; Gibbons, G.W.; Gundlach, C. Dark Matter, Time Varying G, and a Dilaton Field. Phys. Rev. Lett. 1990, 64, 123-126. [CrossRef]

149. Sahni, V.; Shtanov, Y. Brane world models of dark energy. JCAP 2003, 311, 014. [CrossRef]

150. Torres, D.F. Quintessence, superquintessence and observable quantities in Brans-Dicke and nonminimally coupled theories. Phys. Rev. D 2002, 66, 043522. [CrossRef]

151. Chung, D.J.H.; Everett, L.L.; Riotto, A. Quintessence and the underlying particle physics theory. Phys. Lett. B 2003, 556, 61-70. [CrossRef]

152. Faraoni, V. Possible end of the universe in a finite future from dark energy with w-1. Phys. Rev. D 2003, 68, 063508. [CrossRef]

153. Carroll, S.M.; De Felice, A.; Trodden, M. Can we be tricked into thinking that w is less than -1 ? Phys. Rev. D 2005, 71, 023525. [CrossRef]

154. Das, S.; Corasaniti, P.S.; Khoury, J. Super-acceleration as signature of dark sector interaction. Phys. Rev. D 2006, 73, 083509. [CrossRef]

155. Jhingan, S.; Nojiri, S.; Odintsov, S.D.; Sami, M.; Thongkool, I.; Zerbini, S. Phantom and non-phantom dark energy: The Cosmological relevance of non-locally corrected gravity. Phys. Lett. B 2008, 663, 424-428. [CrossRef]

156. Nojiri, S.; Saridakis, E.N. Phantom without ghost. Astrophys. Space Sci. 2013, 347, 221-226. [CrossRef]

157. Ludwick, K.J. Examining the Viability of Phantom Dark Energy. Phys. Rev. D 2015, 92, 063019. [CrossRef] 
158. Cognola, G.; Myrzakulov, R.; Sebastiani, L.; Vagnozzi, S.; Zerbini, S. Covariant Horava-like and mimetic Horndeski gravity: Cosmological solutions and perturbations. Class. Quant. Grav. 2016, 33, 225014. [CrossRef]

159. Sebastiani, L.; Vagnozzi, S.; Myrzakulov, R. Mimetic gravity: A review of recent developments and applications to cosmology and astrophysics. Adv. High Energy Phys. 2017, 2017, 3156915. [CrossRef]

160. Ludwick, K.J. The viability of phantom dark energy: A review. Mod. Phys. Lett. A 2017, 32, 1730025. [CrossRef]

161. Barenboim, G.; Kinney, W.H.; Morse, M.J.P. Phantom Dirac-Born-Infeld Dark Energy. Phys. Rev. D 2018, 98, 083531. [CrossRef]

162. Casalino, A.; Rinaldi, M.; Sebastiani, L.; Vagnozzi, S. Mimicking dark matter and dark energy in a mimetic model compatible with GW170817. Phys. Dark Univ. 2018, 22, 108. [CrossRef]

163. Capozziello, S.; Ruchika.; Sen, A.A. Model independent constraints on dark energy evolution from low-redshift observations. Mon. Not. R. Astron. Soc. 2019, 484, 4484. [CrossRef]

164. Wang, Y.; Pogosian, L.; Zhao, G.B.; Zucca, A. Evolution of dark energy reconstructed from the latest observations. Astrophys. J. 2018, 869, L8. [CrossRef]

165. Dutta, K.; Ruchika.; Roy, A.; Sen, A.A.; Sheikh-Jabbari, M.M. Beyond $\Lambda$ CDM with Low and High Redshift Data: Implications for Dark Energy. arXiv 2018, arXiv:1808.06623.

166. Maldacena, J.M.; Nunez, C. Supergravity description of field theories on curved manifolds and a no go theorem. Int. J. Mod. Phys. A 2001, 16, 822-855. [CrossRef]

167. Silverstein, E. Simple de Sitter Solutions. Phys. Rev. D 2008, 77, 106006. [CrossRef]

168. Danielsson, U.H.; Haque, S.S.; Shiu, G.; Van Riet, T. Towards Classical de Sitter Solutions in String Theory. JHEP 2009, 9, 114. [CrossRef]

169. Wrase, T.; Zagermann, M. On Classical de Sitter Vacua in String Theory. Fortschritte Phys. 2010, 58, $906-910$. [CrossRef]

170. Danielsson, U.H.; Koerber, P.; Van Riet, T. Universal de Sitter solutions at tree-level. JHEP 2010, 5, 090. [CrossRef]

171. Danielsson, U.H.; Haque, S.S.; Koerber, P.; Shiu, G.; Van Riet, T.; Wrase, T. De Sitter hunting in a classical landscape. Fortschritte Phys. 2011, 59, 897-933. [CrossRef]

172. Chen, X.; Shiu, G.; Sumitomo, Y.; Tye, S.H.H. A Global View on The Search for de-Sitter Vacua in (type IIA) String Theory. JHEP 2012, 4, 026. [CrossRef]

173. Danielsson, U.H.; Shiu, G.; Van Riet, T.; Wrase, T. A note on obstinate tachyons in classical dS solutions. JHEP 2013, 3, 138. [CrossRef]

174. Dasgupta, K.; Gwyn, R.; McDonough, E.; Mia, M.; Tatar, R. de Sitter Vacua in Type IIB String Theory: Classical Solutions and Quantum Corrections. JHEP 2014, 7, 054. [CrossRef]

175. Cicoli, M.; De Alwis, S.; Maharana, A.; Muia, F.; Quevedo, F. De Sitter vs Quintessence in String Theory. Fortschritte Phys. 2019, 67, 1800079. [CrossRef]

176. Vafa, C. The String landscape and the swampland. arXiv 2005, arXiv:0509212.

177. Danielsson, U.H.; Van Riet, T. What if string theory has no de Sitter vacua? Int. J. Mod. Phys. 2018, 27, 1830007. [CrossRef]

178. Obied, G.; Ooguri, H.; Spodyneiko, L.; Vafa, C. De Sitter Space and the Swampland. arXiv 2018, arXiv:1806.08362.

179. Ooguri, H.; Palti, E.; Shiu, G.; Vafa, C. Distance and de Sitter Conjectures on the Swampland. Phys. Lett. B 2019, 788, 180-184. [CrossRef]

180. Palti, E. The Swampland: Introduction and Review. Fortschritte Phys. 2019, 67, 1900037. [CrossRef]

181. Agrawal, P.; Obied, G.; Steinhardt, P.J.; Vafa, C. On the Cosmological Implications of the String Swampland. Phys. Lett. B 2018, 784, 271-276. [CrossRef]

182. Andriot, D. On the de Sitter swampland criterion. Phys. Lett. B 2018, 785, 570-573. [CrossRef]

183. Achúcarro, A.; Palma, G.A. The string swampland constraints require multi-field inflation. JCAP 2019, 1902, 041. [CrossRef]

184. Garg, S.K.; Krishnan, C. Bounds on Slow Roll and the de Sitter Swampland. arXiv 2018, arXiv:1807.05193.

185. Kehagias, A.; Riotto, A. A note on Inflation and the Swampland. Fortschritte Phys. 2018, 66, 1800052. [CrossRef] 
186. Heisenberg, L.; Bartelmann, M.; Brandenberger, R.; Refregier, A. Dark Energy in the Swampland. Phys. Rev. D 2018, 98, 123502. [CrossRef]

187. Kinney, W.H.; Vagnozzi, S.; Visinelli, L. The zoo plot meets the swampland: Mutual (in)consistency of single-field inflation, string conjectures, and cosmological data. Class. Quant. Grav. 2019, 36, 117001. [CrossRef]

188. Akrami, Y.; Kallosh, R.; Linde, A.; Vardanyan, V. The Landscape, the Swampland and the Era of Precision Cosmology. Fortschritte Phys. 2019, 67, 1800075. [CrossRef]

189. Murayama, H.; Yamazaki, M.; Yanagida, T.T. Do We Live in the Swampland? JHEP 2018, 12, 032. [CrossRef]

190. Kinney, W.H. Eternal Inflation and the Refined Swampland Conjecture. Phys. Rev. Lett. 2019, 122, 081302. [CrossRef]

191. Kachru, S.; Kallosh, R.; Linde, A.D.; Trivedi, S.P. De Sitter vacua in string theory. Phys. Rev. D 2003, 68, 046005. [CrossRef]

192. Maldacena, J.M. The Large N limit of superconformal field theories and supergravity. Int. J. Theor. Phys. 1999, 38, 1113-1133, doi:10.1023/A:1026654312961. [CrossRef]

193. Hartle, J.B.; Hawking, S.W.; Hertog, T. Accelerated Expansion from Negative $\Lambda$. arXiv 2012, arXiv:1205.3807.

194. Svrcek, P.; Witten, E. Axions In String Theory. JHEP 2006, 6, 051. [CrossRef]

195. Arvanitaki, A.; Dimopoulos, S.; Dubovsky, S.; Kaloper, N.; March-Russell, J. String Axiverse. Phys. Rev. D 2010, 81, 123530. [CrossRef]

196. Arvanitaki, A.; Dubovsky, S. Exploring the String Axiverse with Precision Black Hole Physics. Phys. Rev. D 2011, 83, 044026. [CrossRef]

197. Cicoli, M.; Goodsell, M.; Ringwald, A. The type IIB string axiverse and its low-energy phenomenology. JHEP 2012, 10, 146. [CrossRef]

198. Conlon, J.P.; Marsh, M.C.D. The Cosmophenomenology of Axionic Dark Radiation. JHEP 2013, 10, 214. [CrossRef]

199. Cicoli, M.; Conlon, J.P.; Marsh, M.C.D.; Rummel, M. $3.55 \mathrm{keV}$ photon line and its morphology from a $3.55 \mathrm{keV}$ axionlike particle line. Phys. Rev. D 2014, 90, 023540. [CrossRef]

200. Marsh, D.J.E. Axion Cosmology. Phys. Rept. 2016, 643, 1-79. [CrossRef]

201. Visinelli, L. Light axion-like dark matter must be present during inflation. Phys. Rev. D 2017, 96, 023013. [CrossRef]

202. Poulin, V.; Smith, T.L.; Grin, D.; Karwal, T.; Kamionkowski, M. Cosmological implications of ultralight axionlike fields. Phys. Rev. D 2018, 98, 083525. [CrossRef]

203. Kitajima, N.; Soda, J.; Urakawa, Y. Gravitational wave forest from string axiverse. JCAP 2018, $1810,008$. [CrossRef]

204. Visinelli, L.; Vagnozzi, S. Cosmological window onto the string axiverse and the supersymmetry breaking scale. Phys. Rev. D 2019, 99, 063517. [CrossRef]

205. Odintsov, S.D.; Oikonomou, V.K. $f(R)$ Gravity Inflation with String-Corrected Axion Dark Matter. Phys. Rev. D 2019, 99, 064049. [CrossRef]

206. Odintsov, S.D.; Oikonomou, V.K. Unification of Inflation with Dark Energy in $f(R)$ Gravity and Axion Dark Matter. Phys. Rev. D 2019, 99, 104070. [CrossRef]

207. Ramberg, N.; Visinelli, L. Probing the Early Universe with Axion Physics and Gravitational Waves. Phys. Rev. D 2019, 99, 123513. [CrossRef]

208. Reynolds, C.S.; Marsh, M.C.D.; Russell, H.R.; Fabian, A.C.; Smith, R.N.; Tombesi, F. Astrophysical limits on very light axion-like particles from Chandra grating spectroscopy of NGC 1275. arXiv 2019, arXiv:1907.05475.

209. Beutler, F.; Blake, C.; Colless, M.; Jones, D.H.; Staveley-Smith, L.; Campbell, L.; Parker, Q.; Saunders, W.; Watson, F. The 6dF Galaxy Survey: Baryon Acoustic Oscillations and the Local Hubble Constant. Mon. Not. R. Astron. Soc. 2011, 416, 3017-3032. [CrossRef]

210. Ross, A.J.; Samushia, L.; Howlett, C.; Percival, W.J.; Burden, A.; Manera, M. The clustering of the SDSS DR7 main Galaxy sample-I. A 4 per cent distance measure at $z=0.15$. Mon. Not. R. Astron. Soc. 2015, 449, 835-847. [CrossRef]

211. Des, Bourboux, H.D.M.; Le Goff, J.M.; Blomqvist, M.; Guy, J.; Rich, J.; Yèche, C. Baryon acoustic oscillations from the complete SDSS-III Ly $\alpha$-quasar cross-correlation function at $z=2.4$. Astron. Astrophys. 2017, 608, A130. [CrossRef] 
212. Bautista, J.E.; Guy, J.; Rich, J.; Blomqvist, M.; Des, Bourboux, H.D.M.; Pieri, M.M. Measurement of baryon acoustic oscillation correlations at $z=2.3$ with SDSS DR12 Ly $\alpha$-Forests. Astron. Astrophys. 2017, 603, A12. [CrossRef]

213. Ata, M.; Baumgarten, F.; Bautista, J.; Beutler, F.; Bizyaev, D.; Blanton, M.R. The clustering of the SDSS-IV extended Baryon Oscillation Spectroscopic Survey DR14 quasar sample: First measurement of baryon acoustic oscillations between redshift 0.8 and 2.2. Mon. Not. R. Astron. Soc. 2018, 473, 4773-4794. [CrossRef]

214. Scolnic, D.M.; Jones, D.O.; Rest, A.; Pan, Y.C.; Chornock, R.; Foley, R.J. The Complete Light-curve Sample of Spectroscopically Confirmed SNe Ia from Pan-STARRS1 and Cosmological Constraints from the Combined Pantheon Sample. Astrophys. J. 2018, 859, 101. [CrossRef]

215. Grande, J.; Sola, J.; Stefancic, H. LXCDM: A Cosmon model solution to the cosmological coincidence problem? JCAP 2006, 608, 011. [CrossRef]

216. Grande, J.; Sola, J.; Stefancic, H. Composite dark energy: Cosmon models with running cosmological term and gravitational coupling. Phys. Lett. B 2007, 645, 236-244. [CrossRef]

217. Guo, Z.K.; Piao, Y.S.; Zhang, X.M.; Zhang, Y.Z. Cosmological evolution of a quintom model of dark energy. Phys. Lett. B 2005, 608, 177-182. [CrossRef]

218. Hu, W. Crossing the phantom divide: Dark energy internal degrees of freedom. Phys. Rev. D 2005, 71, 047301. [CrossRef]

219. Cai, Y.F.; Saridakis, E.N.; Setare, M.R.; Xia, J.Q. Quintom Cosmology: Theoretical implications and observations. Phys. Rept. 2010, 493, 1-60. [CrossRef]

220. Saridakis, E.N.; Weller, J.M. A Quintom scenario with mixed kinetic terms. Phys. Rev. D 2010, 81, 123523. [CrossRef]

221. Saridakis, E.N.; Tsoukalas, M. Cosmology in new gravitational scalar-tensor theories. Phys. Rev. D 2016, 93, 124032. [CrossRef]

222. Amendola, L.; Tsujikawa, S. Phantom crossing, equation-of-state singularities, and local gravity constraints in $\mathrm{f}(\mathrm{R})$ models. Phys. Lett. B 2008, 660, 125-132. [CrossRef]

223. Saridakis, E.N.; Ward, J. Quintessence and phantom dark energy from ghost D-branes. Phys. Rev. D 2009, 80, 083003. [CrossRef]

224. Wu, P.; Yu, H.W. $f(T)$ models with phantom divide line crossing. Eur. Phys. J. C 2011, 71, 1552. [CrossRef]

225. Elizalde, E.; Odintsov, S.D.; Sebastiani, L.; Zerbini, S. Oscillations of the $F(R)$ dark energy in the accelerating universe. Eur. Phys. J. C 2012, 72, 1843. [CrossRef]

226. Saridakis, E.N. Phantom crossing and quintessence limit in extended nonlinear massive gravity. Class. Quant. Grav. 2013, 30, 075003. [CrossRef]

227. Pan, S.; Chakraborty, S. A Cosmological Study in Massive Gravity theory. Ann. Phys. 2015, 360, $180-193$. [CrossRef]

228. Dhawan, S.; Goobar, A.; Mörtsell, E.; Amanullah, R.; Feindt, U. Narrowing down the possible explanations of cosmic acceleration with geometric probes. JCAP 2017, 1707, 040. [CrossRef]

229. Elizalde, E.; Odintsov, S.D.; Sebastiani, L.; Myrzakulov, R. Beyond-one-loop quantum gravity action yielding both inflation and late-time acceleration. Nucl. Phys. B 2017, 921, 411-435. [CrossRef]

230. Odintsov, S.D.; Oikonomou, V.K.; Sebastiani, L. Unification of Constant-roll Inflation and Dark Energy with Logarithmic $R^{2}$-corrected and Exponential $F(R)$ Gravity. Nucl. Phys. B 2017, 923, 608-632. [CrossRef]

231. Dutta, J.; Khyllep, W.; Saridakis, E.N.; Tamanini, N.; Vagnozzi, S. Cosmological dynamics of mimetic gravity. JCAP 2018, 1802, 041. [CrossRef]

232. Amendola, L. Coupled quintessence. Phys. Rev. D 2000, 62, 043511. [CrossRef]

233. Barrow, J.D.; Clifton, T. Cosmologies with energy exchange. Phys. Rev. D 2006, 73, 103520. [CrossRef]

234. He, J.H.; Wang, B. Effects of the interaction between dark energy and dark matter on cosmological parameters. JCAP 2008, 806, 010. [CrossRef]

235. Valiviita, J.; Majerotto, E.; Maartens, R. Instability in interacting dark energy and dark matter fluids. JCAP 2008, 807, 020. [CrossRef]

236. Gavela, M.B.; Hernandez, D.; Lopez Honorez, L.; Mena, O.; Rigolin, S. Dark coupling. JCAP 2009, $907,034$. [CrossRef]

237. Martinelli, M.; Lopez Honorez, L.; Melchiorri, A.; Mena, O. Future CMB cosmological constraints in a dark coupled universe. Phys. Rev. D 2010, 81, 103534. [CrossRef] 
238. Pan, S.; Bhattacharya, S.; Chakraborty, S. An analytic model for interacting dark energy and its observational constraints. Mon. Not. R. Astron. Soc. 2015, 452, 3038-3046. [CrossRef]

239. Tamanini, N. Phenomenological models of dark energy interacting with dark matter. Phys. Rev. D 2015, 92, 043524. [CrossRef]

240. Nunes, R.C.; Pan, S.; Saridakis, E.N. New constraints on interacting dark energy from cosmic chronometers. Phys. Rev. D 2016, 94, 023508. [CrossRef]

241. Pan, S.; Sharov, G.S. A model with interaction of dark components and recent observational data. Mon. Not. R. Astron. Soc. 2017, 472, 4736-4749. [CrossRef]

242. Sharov, G.S.; Bhattacharya, S.; Pan, S.; Nunes, R.C.; Chakraborty, S. A new interacting two fluid model and its consequences. Mon. Not. R. Astron. Soc. 2017, 466, 3497-3506. [CrossRef]

243. Pan, S.; Mukherjee, A.; Banerjee, N. Astronomical bounds on a cosmological model allowing a general interaction in the dark sector. Mon. Not. R. Astron. Soc. 2018, 477, 1189-1205. [CrossRef]

244. Yang, W.; Banerjee, N.; Paliathanasis, A.; Pan, S. Reconstructing the dark matter and dark energy interaction scenarios from observations. arXiv 2018, arXiv:1812.06854.

245. Paliathanasis, A.; Pan, S.; Yang, W. Dynamics of nonlinear interacting dark energy models. arXiv 2019, arXiv:1903.02370.

246. Yang, W.; Pan, S.; Di Valentino, E.; Wang, B.; Wang, A. Forecasting Interacting Vacuum-Energy Models using Gravitational Waves. arXiv 2019, arXiv:1904.11980.

247. Lima, J.A.S.; Silva, F.E.; Santos, R.C. Accelerating Cold Dark Matter Cosmology $\left(\Omega_{\Lambda} \equiv 0\right)$. Class. Quant. Grav. 2008, 25, 205006. [CrossRef]

248. Lima, J.A.S.; Jesus, J.F.; Oliveira, F.A. CDM Accelerating Cosmology as an Alternative to LCDM model. JCAP 2010, 1011, 027. [CrossRef]

249. Pan, S.; Chakraborty, S. Will There Be Future Deceleration? A Study of Particle Creation Mechanism in Nonequilibrium Thermodynamics. Adv. High Energy Phys. 2015, 2015, 654025. [CrossRef]

250. Nunes, R.C.; Pavón, D. Phantom behavior via cosmological creation of particles. Phys. Rev. D 2015, 91, 063526. [CrossRef]

251. de Haro, J.; Pan, S. Gravitationally induced adiabatic particle production: From Big Bang to de Sitter. Class. Quant. Gravity 2016, 33, 165007. [CrossRef]

252. Pan, S.; de Haro, J.; Paliathanasis, A.; Slagter, R.J. Evolution and Dynamics of a Matter creation model. Mon. Not. R. Astron. Soc. 2016, 460, 1445-1456. [CrossRef]

253. Nunes, R.C.; Pan, S. Cosmological consequences of an adiabatic matter creation process. Mon. Not. R. Astron. Soc. 2016, 459, 673-682. [CrossRef]

254. Pan, S.; Kumar Pal, B.; Pramanik, S. Gravitationally influenced particle creation models and late-time cosmic acceleration. Int. J. Geom. Meth. Mod. Phys. 2017, 15, 1850042. [CrossRef]

255. Paliathanasis, A.; Barrow, J.D.; Pan, S. Cosmological solutions with gravitational particle production and nonzero curvature. Phys. Rev. D 2017, 95, 103516. [CrossRef]

256. Pan, S.; Barrow, J.D.; Paliathanasis, A. Two-fluid solutions of particle-creation cosmologies. Eur. Phys. J. C 2019, 79, 115. [CrossRef]

257. Vikman, A. Can dark energy evolve to the phantom? Phys. Rev. D 2005, 71, 023515. [CrossRef]

258. Creminelli, P.; D'Amico, G.; Norena, J.; Vernizzi, F. The Effective Theory of Quintessence: The w $<-1$ Side Unveiled. JCAP 2009, 902, 018. [CrossRef]

259. Deffayet, C.; Pujolas, O.; Sawicki, I.; Vikman, A. Imperfect Dark Energy from Kinetic Gravity Braiding. JCAP 2010, 1010, 026. [CrossRef]

260. Akrami, Y.; Kallosh, R.; Linde, A.; Vardanyan, V. Dark energy, $\alpha$-attractors, and large-scale structure surveys. JCAP 2018, 1806, 041. [CrossRef]

261. Paliathanasis, A.; Leon, G.; Pan, S. Exact Solutions in Multi-Scalar Field Cosmology. arXiv 2018, arXiv:1811.10038.

262. Peebles, P.J.E.; Yu, J.T. Primeval adiabatic perturbation in an expanding universe. Astrophys. J. 1970, 162, 815-836. [CrossRef]

263. Sunyaev, R.A.; Zeldovich, Ya.B. Small scale fluctuations of relic radiation. Astrophys. Space Sci. 1970, 7, 3-19.

264. Sunyaev, R.A.; Zeldovich, Ya.B. The Interaction of matter and radiation in the hot model of the universe. Astrophys. Space Sci. 1970, 7, 20-30. 
265. Eisenstein, D.J.; Zehavi, I.; Hogg, D.W.; Scoccimarro, R.; Blanton, M.R.; Nichol, R.C. Detection of the Baryon Acoustic Peak in the Large-Scale Correlation Function of SDSS Luminous Red Galaxies. Astrophys. J. 2005, 633, 560-574. [CrossRef]

266. Aubourg, É; Bailey, S.; Bautista, J.E.; Beutler, F.; Bhardwaj, V.; Bizyaev, D. Cosmological implications of baryon acoustic oscillation measurements. Phys. Rev. D 2015, 92, 123516. [CrossRef]

267. Cole, S.; Percival, W.J.; Peacock, J.A.; Norberg, P.; Baugh, C.M.; Frenk, C.S. The 2dF Galaxy Redshift Survey: Power-spectrum analysis of the final dataset and cosmological implications. Mon. Not. R. Astron. Soc. 2005, 362, 505-534. [CrossRef]

268. Yang, W.; Vagnozzi, S.; Di Valentino, E.; Nunes, R.C.; Pan, S.; Mota, D.F. Listening to the sound of dark sector interactions with gravitational wave standard sirens. arXiv 2019, arXiv:1905.08286.

269. Betoule, M.; Kessler, R.; Guy, J.; Mosher, J.; Hardin, D.; Biswas, R. Improved cosmological constraints from a joint analysis of the SDSS-II and SNLS supernova samples. Astron. Astrophys. 2014, 568, A22. [CrossRef]

270. Riess, A.G.; Strolger, L.G.; Casertano, S.; Ferguson, H.C.; Mobasher, B.; Gold, B. New Hubble Space Telescope Discoveries of Type Ia Supernovae at $\mathrm{z}>=1$ : Narrowing Constraints on the Early Behavior of Dark Energy. Astrophys. J. 2007, 659, 98-121. [CrossRef]

271. Hicken, M.; Challis, P.; Jha, S.; Kirshner, R.P.; Matheson, T.; Modjaz, M.; Rest, A.; Wood-Vasey, W.M.; Bakos, G.; Barton, E.J. CfA3: 185 Type Ia Supernova Light Curves from the CfA. Astrophys. J. 2009, 700, 331-357. [CrossRef]

272. Conley, A.; Guy, J.; Sullivan, M.; Regnault, N.; Astier, P.; Balland, C.; Basa, S.; Carlberg, R.G.; Fouchez, D.; Hardin, D. Supernova Constraints and Systematic Uncertainties from the First Three Years of the Supernova Legacy Survey. Astrophys. J. Suppl. 2011, 192, 1. [CrossRef]

273. Suzuki, N.; Rubin, D.; Lidman, C.; Aldering, G.; Amanullah, R.; Barbary, K.; Barrientos, L.F.; Botyanszki, J.; Brodwin, M.; Connolly, N. The Hubble Space Telescope Cluster Supernova Survey. V. Improving the Dark-energy Constraints above z \&gt; 1 and Building an Early-type-hosted Supernova Sample. Astrophys. J. 2012, 746, 85. [CrossRef]

274. Sako, M.; Bassett, B.; Becker, A.C.; Brown, P.J.; Campbell, H.; Wolf, R. The Data Release of the Sloan Digital Sky Survey-II Supernova Survey. Publ. Astron. Soc. Pac. 2018, 130, 064002. [CrossRef]

275. Jones, D.O.; Scolnic, D.M.; Riess, A.G.; Rest, A.; Kirshner, R.P.; Berger, E. Measuring Dark Energy Properties with Photometrically Classified Pan-STARRS Supernovae. II. Cosmological Parameters. Astrophys. J. 2018, 857, 51. [CrossRef]

276. Cuesta, A.J.; Verde, L.; Riess, A.; Jimenez, R. Calibrating the cosmic distance scale ladder: The role of the sound horizon scale and the local expansion rate as distance anchors. Mon. Not. R. Astron. Soc. 2015, 448, 3463-3471. [CrossRef]

277. Meng, X.L.; Wang, X.; Li, S.Y.; Zhang, T.J. Utility of observational Hubble parameter data on dark energy evolution. arXiv 2015, arXiv:1507.02517.

278. Moresco, M.; Pozzetti, L.; Cimatti, A.; Jimenez, R.; Maraston, C.; Verde, L.; Thomas, D.; Citro, A.; Tojeiro, R.; Wilkinson, D. A $6 \%$ measurement of the Hubble parameter at $z \sim 0.45$ : Direct evidence of the epoch of cosmic re-acceleration. JCAP 2016, 1605, 014. [CrossRef]

279. Kosowsky, A.; Milosavljevic, M.; Jimenez, R. Efficient cosmological parameter estimation from microwave background anisotropies. Phys. Rev. D 2002, 66, 063007. [CrossRef]

280. Wang, Y.; Mukherjee, P. Observational Constraints on Dark Energy and Cosmic Curvature. Phys. Rev. D 2007, 76, 103533. [CrossRef]

281. Fixsen, D.J.; Cheng, E.S.; Gales, J.M.; Mather, J.C.; Shafer, R.A.; Wright, E.L. The Cosmic Microwave Background spectrum from the full COBE FIRAS data set. Astrophys. J. 1996, 473, 576. [CrossRef]

282. de Salas, P.F.; Forero, D.V.; Ternes, C.A.; Tortola, M.; Valle, J.W.F. Status of neutrino oscillations 2018: $3 \sigma$ hint for normal mass ordering and improved CP sensitivity. Phys. Lett. B 2018, 782, 633-640. [CrossRef]

283. Vagnozzi, S.; Giusarma, E.; Mena, O.; Freese, K.; Gerbino, M.; Ho, S.; Lattanzi, M. Unveiling $v$ secrets with cosmological data: Neutrino masses and mass hierarchy. Phys. Rev. D 2017, 96, 123503. [CrossRef]

284. Simpson, F.; Jimenez, R.; Pena-Garay, C.; Verde, L. Strong Bayesian Evidence for the Normal Neutrino Hierarchy. JCAP 2017, 1706, 029. [CrossRef]

285. Schwetz, T.; Freese, K.; Gerbino, M.; Giusarma, E.; Hannestad, S.; Lattanzi, M.; Mena, O.; Vagnozzi, S. Comment on "Strong Evidence for the Normal Neutrino Hierarchy". arXiv 2017, arXiv:1703.04585. 
286. De Salas, P.F.; Gariazzo, S.; Mena, O.; Ternes, C.A.; Tórtola, M. Neutrino Mass Ordering from Oscillations and Beyond: 2018 Status and Future Prospects. Front. Astron. Space Sci. 2018, 5, 36. [CrossRef]

287. Palanque-Delabrouille, N.; Yèche, C.; Baur, J.; Magneville, C.; Rossi, G.; Lesgourgues, J. Neutrino masses and cosmology with Lyman-alpha forest power spectrum. JCAP 2015, 1511, 011. [CrossRef]

288. Visinelli, L. Neutrino flavor oscillations in a curved space-time. Gen. Relat. Gravit. 2015, 47, 62. [CrossRef]

289. Cuesta, A.J.; Niro, V.; Verde, L. Neutrino mass limits: Robust information from the power spectrum of galaxy surveys. Phys. Dark Univ. 2016, 13, 77-86. [CrossRef]

290. Huang, Q.G.; Wang, K.; Wang, S. Constraints on the neutrino mass and mass hierarchy from cosmological observations. Eur. Phys. J. C 2016, 76, 489. [CrossRef]

291. Giusarma, E.; Gerbino, M.; Mena, O.; Vagnozzi, S.; Ho, S.; Freese, K. Improvement of cosmological neutrino mass bounds. Phys. Rev. D 2016, 94, 083522. [CrossRef]

292. Giusarma, E.; Vagnozzi, S.; Ho, S.; Ferraro, S.; Freese, K.; Kamen-Rubio, R.; Luk, K.B. Scale-dependent galaxy bias, CMB lensing-galaxy cross-correlation, and neutrino masses. Phys. Rev. D 2018, 98, 123526. [CrossRef]

293. Vagnozzi, S. Cosmological searches for the neutrino mass scale and mass ordering. arXiv 2019, arXiv:1907.08010.

294. Foreman-Mackey, D.; Hogg, D.W.; Lang, D.; Goodman, J. emcee: The MCMC Hammer. Publ. Astron. Soc. Pac. 2013, 125, 306-312. [CrossRef]

295. Akaike, H. A new look at the statistical model identification. IEEE Trans. Autom. Control 1974, 19, 716-723. [CrossRef]

296. Lemos, P.; Lee, E.; Efstathiou, G.; Gratton, S. Model independent $H(z)$ reconstruction using the cosmic inverse distance ladder. Mon. Not. R. Astron. Soc. 2019, 483, 4803-4810. [CrossRef]

297. Ade, P.; Aguirre, J.; Ahmed, Z.; Aiola, S.; Ali, A.; Alonso, D. The Simons Observatory: Science goals and forecasts. JCAP 2019, 1902, 056. [CrossRef]

298. Abitbol, M.H.; Adachi, S.; Ade, P.; Aguirre, J.; Ahmed, Z.; Aiola, S. The Simons Observatory: Astro2020 Decadal Project Whitepaper. arXiv 2019, arXiv:1907.08284.

299. Abazajian, K.N.; Adshead, P.; Ahmed, Z.; Allen, S.W.; Alonso, D.; Arnold, K.S. CMB-S4 science book. arXiv 2016, arXiv:1610.02743.

300. Du, M.; Yang, W.; Xu, L.; Pan, S.; Mota, D.F. Future Constraints on Dynamical Dark-Energy using Gravitational-Wave Standard Sirens. arXiv 2018, arXiv:1812.01440.

(C) 2019 by the authors. Licensee MDPI, Basel, Switzerland. This article is an open access article distributed under the terms and conditions of the Creative Commons Attribution (CC BY) license (http://creativecommons.org/licenses/by/4.0/). 